\title{
Apuntes para la comprensión de "El descubrimiento de Harry", de Matthew Lipman*
}

\author{
Diego Antonio Pineda R.
}

\begin{abstract}
Recibido: 13 de septiembre de 2014 - Revisado: 5 de noviembre de 2014 -
\end{abstract} Aprobado: 12 de diciembre de 2014

Este trabajo recoge algunas ideas generales en torno a la novela de Matthew Lipman El descubrimiento de Harry. La pretensión del artículo es ofrecer "claves de lectura" que ayuden a los maestros que leen la novela, o la trabajan con su grupo de alumnos en un salón de clase, a comprender su significado filosófico. Para ello el artículo describe el contexto definido por las cuatro novelas del programa completo de "Filosofía para niños" que propone el autor y, a partir de allí, aborda cinco elementos que resultan orientadores para el lector potencial: cuál es el descubrimiento de Harry, la estructura teórica y narrativa de la novela, la relación de la lógica con otros problemas filosóficos, las "formas del pensamiento superior", y algunas sugerencias para trabajar la novela en el aula de clase. Recorre en el proceso aspectos propios de la filosofía que la novela asume, como las proposiciones categóricas, que constituyen el principal descubrimiento de Harry, la construcción del lenguaje de la lógica formal, los actos mentales y los mecanismos de validación de los razonamientos, así como la relación que se da entre pensamiento y lenguaje, y sus implicaciones en el abordaje de problemas desde los diversos ámbitos de la filosofía: cosmológicos, políticos, estéticos, epistemológicos, éticos, etc.

Palabras clave: Filosofía, Filosofía para Niños (FpN), Lipman, lógica formal, modelos de pensamiento.

Artículo de reflexión que asume como punto de partida el quehacer del autor como traductor autorizado de los trabajos de Lipman para Colombia y en la investigación en $\mathrm{FpN}$

** PhD en Filosofía de la Universidad Javeriana, institución en la que se desempeña actualmente como Decano de la Facultad de Filosofía. Dirección postal: Carrera 7 40-62, Bogotá, Colombia. Correo electrónico: diegoantpineda@yahoo. com, diegopi@javeriana.edu.co. 


\title{
Notes for understanding "Harry Stottlemeier's Discovery "by Matthew Lipman*
}

\author{
Diego Antonio Pineda R.*
}

This paper covers some general ideas about the novel written by Matthew Lipman: Harry Stottlemeier's Discovery. The aim of the paper is to offer "reading keys" that help teachers reading the novel, or teachers that are using it with their students' group in class, to understand its philosophical meaning. To do that I describe the context defined by the four novels of the complete program of "Philosophy for Children" proposed by the author and, from there, I address five guiding elements for the potential reader: The discovery of Harry, the theoretical structure and narrative of the novel, the relationship between logic and other philosophical problems, the "forms of the superior thinking", and some suggestions in order to work the novel in class. The article deals with philosophical aspects that the novel assumes, as categorical propositions, which are the main discovery of Harry, language construction of formal logic, mental acts and reasoning validation mechanisms, as well as the relationship that exists between thought and language, and its implications in addressing problems from the different areas of philosophy: cosmological, political, aesthetic, epistemological, ethical, etc.

Keywords: Philosophy, Philosophy for Children (Philosophy for Children), Lipman, formal logic, thinking models.

\footnotetext{
* Reflection article that takes as its starting point the work of the author and translator of the works authorized Lipman for Colombia and Philosophy for research

** PhD in philosophy at the Javeriana University, an institution where he currently serves as Dean of the Faculty of Philosophy. Address: Carrera 7 40-62, Bogota, Colombia. Email: diegoantpineda @ yahoo.com, diegopi@javeriana.edu.co.
} 


\title{
Notes pour la compréhension de "La découverte d'Harry", de Matthew Lipman*
}

\author{
Diego Antonio Pineda R.
}

Ce travail reprend certaines idées générales autour du roman de Matthew Lipman La découverte d'Harry. La prétention de l'article es d'offrir des «clés de lecture» qui aident aux enseignants qui lissent le roman, ou qui le travaillent avec ses groupes d'élèves dans une salle de classe, à comprendre son sens philosophique. Pour cela, l'article décrit le contexte définit par les quatre romans du programme complet de «Philosophie pour Enfants» qui propose l'auteur et, à partir de là, il aborde cinq éléments qui orientent le lecteur potentiel: la découverte d'Harry, la structure théorique et narrative du roman, la relation de la logique avec d'autres problèmes philosophiques, les «formes de la pensée supérieure», et quelques suggestions pour travailler le roman dans la salle de classe. Finalement, il présente quelques suggestions pour travailler le roman dans la salle de classe. Il fait un parcours des aspects propres à la philosophie que le roman assume, telles que les propositions catégoriques, qui constituent la découverte fondamentale d'Harry, la construction du langage de la logique formelle, les actes mentaux et les mécanismes de validation des raisonnements, ainsi que la relation existante entre la pensée et le langage, et ses implications dans l'approche de problèmes à partir de divers domaines de la philosophie: cosmologiques, politiques, esthétiques, épistémologiques, éthiques, etc.

Mots clés: Philosophie, Philosophie pour Enfants (PPE), Lipman, logique formelle, schéma de pensée.

L'article de réflexion qui prend comme point de départ le travail de l'auteur et traducteur des oeuvres autorisées Lipman pour la Colombie et la philosophie pour la recherche

** Doctorat en philosophie à l'Université Javeriana, une institution où il est actuellement doyen de la Faculté de Philosophie. Adresse: Carrera 7 40-62, Bogota, Colombie. Email: diegoantpineda @ yahoo.com, diegopi@javeriana.edu.co. 
Con la escritura de El descubrimiento de Harry en 1969 y su puesta en práctica con niños de quinto grado un año más tarde, vio la luz la filosofía como tema y currículo de la escuela primaria. Apareció en escena sin haber sido antes prevista o propuesta, defendida o atacada. Aún las pocas personas que la aceptaron cuando llegó, tenían dudas acerca de lo que podría realizar. Representante de un género hasta entonces inexistente, este libro recibió al comienzo escasa o nula atención y parecía que pronto llegaría a ser superfluo. Al salir, la novela llamó la atención de una parte de un grupo minúsculo de personas. A decir verdad, a muchas de ellas el libro sólo les atraía como curiosidad: lo miraban como uno de esos extraños objetos que se encuentran en la playa o uno de los trabajos menores de Borges. ¿Pero qué es lo que efectivamente veían en él? ¿Qué poder podría otorgar esa novela? ¿Y qué peligros, en ese momento latentes, podría su presencia despertar en la escuela?

Matthew Lipman

En las siguientes páginas presento algunas ideas generales (algunas de ellas, incluso, no muy claramente conectadas entre sí) en torno a la novela de Matthew Lipman El descubrimiento de Harry. Dado el carácter más bien intuitivo de estas reflexiones prefiero llamarlas simplemente "apuntes" (en realidad eso son, unos apuntes personales que he empezado a transcribir para compartir con otros), pues son simplemente ideas para poner en discusión y que no tienen otro fin que lanzar algunas hipótesis que deben ser examinadas por los maestros que leen la novela o la trabajan en un salón de clase con su grupo de alumnos. Estas líneas deben servir especialmente para acercar al texto a sus potenciales lectores con algunas posibles "claves de lectura" que le ayuden a comprender el significado filosófico de la novela.

Cada novela del programa "Filosofía para niños" (en adelante simplemente FpN), junto con su correspondiente manual para el maestro, constituye un programa completo. De esta manera:

a. Cada una es una introducción a la investigación de alguna problemática filosófica específica. Así, por ejemplo:

- El descubrimiento de Harry (en adelante simplemente DH) es una introducción a la lógica formal.

- Elisa es una introducción a la investigación ética.

- Susy es una introducción a la problemática estética, con un énfasis en la relación entre pensamiento y escritura (especialmente la escritura poética).

- Marcos es una introducción a la investigación filosófica sobre la vida social y política. 
b. Los 4 textos previamente señalados forman una secuencia:

- Tanto narrativa (son los mismos personajes -el grupo de Harry y sus amigos Elisa, Toño, Marcos, Susy, Ana María, Guillermo, etc. Junto con sus respectivos padres y los profesores del colegio al que pertenecen-y estos personajes se van desenvolviendo en un ambiente similar: el de su colegio, sus familias y los grupos de amigos),

- como filosófica, pues hay una articulación interna entre los cuatro programas, que va desde el descubrimiento de las "reglas del pensamiento" (lógica formal) en DH hasta su aplicación en distintos contextos a problemas de diversa índole: éticos, metafísicos, políticos, estéticos, epistemológicos, de filosofía de la educación, de la ciencia, del lenguaje, etc.

c. A lo largo de toda esta secuencia, sin embargo, el núcleo de toda la "trama filosófica" desarrollada está en el descubrimiento de las "reglas del pensar", que se inicia en $\mathrm{DH}$, pero que se continúa a lo largo de los demás textos. Este descubrimiento de las "reglas del pensar" (que, en cierto sentido, equivalen a las reglas de la lógica formal) prepara a los niños que trabajan con este texto para:

- El descubrimiento de la forma de las proposiciones lógicas.

- El examen de las reglas que permiten construir argumentos correctos.

- La elaboración, a partir de lo anterior, de un "lenguaje del pensamiento" que los guiará a lo largo de todas sus indagaciones posteriores.

d. Por lo tanto, debemos considerar DH como el "origen" de todo el programa FpN, pues es precisamente en este texto que se realiza el "descubrimiento" fundamental: que, al pensar en cualquier cosa que se nos presente, seguimos algunas reglas generales, y que, si estamos atentos a ellas, encontraremos en estas reglas una herramienta esencial en la construcción de mejores juicios, puesto que podemos desarrollar el hábito de pensar de un modo ordenado, sistemático y coherente.

Esto último es lo que hace tan fundamental el trabajar $\mathrm{DH}$ con los niños y/o jóvenes a quienes pretendemos desarrollar una capacidad para pensar filosóficamente. Por ello, a pesar de muchas de las dificultades efectivas que plantea el trabajar con un texto como éste (dificultades que han conducido a que, en algunos países, simplemente se haya suprimido este texto como parte de su proyecto de trabajo en $\mathrm{FpN}$ ), considero que el trabajo con $\mathrm{DH}$ es simplemente insustituible, por lo menos mientras no encontremos o desarrollemos una mejor propuesta que la de M. Lipman.

En estos "apuntes" me referiré a varios asuntos que buscan ayudar a que el lector potencial de DH (niño o joven, maestro, padre de familia) entienda mejor el sentido filosófico y las virtudes pedagógicas de un texto como éste. 
Me referiré, entonces, a cinco puntos básicos:

1. ¿Cuál fue el "descubrimiento" de Harry?

2. La estructura teórica y narrativa de DH.

3. La relación de la lógica con otros problemas filosóficos.

4. Las "formas del pensamiento superior" en DH.

5. Algunas sugerencias para el trabajo con $\mathrm{DH}$ en el aula de clase.

\section{1. ¿Cuál fue el "descubrimiento" de Harry?}

A primera vista (esto es, si nos quedamos exclusivamente con la historia que se nos cuenta en este texto, sin ahondar en su sentido filosófico) el "descubrimiento" de Harry fue muy simple, casi trivial: que en las oraciones que empiezan por todos, cuando invertimos el sujeto y el predicado, si son verdaderas, se convierten en falsas; mientras que, en las oraciones que empiezan por ningún, al invertir el sujeto y el predicado, la oración conserva su valor de verdad (si son verdaderas siguen siendo verdaderas, $\mathrm{y}$, si son falsas, continúan siendo falsas) (cf. Capítulo Primero) ${ }^{1}$.

Lo que descubre Harry en este primer capítulo es, sin embargo, algo más profundo que esto: lo que descubre es el mecanismo de la conversión de las proposiciones categóricas. ¿En qué consiste esto? En el hecho de que cierto tipo de proposiciones (las proposiciones universales afirmativas, o proposiciones en A) no admiten esta inversión, pues la inferencia que en estos casos se hace es incorrecta. Así, por ejemplo: de la proposición "todos los leones son animales" no se puede deducir la proposición "todos los animales son leones" (mientras la primera es verdadera, la segunda será falsa). Por el contrario, cuando se trata de proposiciones universales negativas (o proposiciones en E), éstas sí admiten la inversión del sujeto y el predicado. De la proposición "ningún caballo es un león" se puede deducir la proposición "ningún león es un caballo". Este "descubrimiento", que Harry hace en el primer capítulo de una forma más bien intuitiva, sólo podrá expresarlo de una forma más explícita hasta el capítulo doce (cf. pp. 91 y ss).

Es importante no ver este "descubrimiento" como algo aislado, como una "regla" suelta sin mayor valor práctico. Por el contrario, sobre la base de este primer "descubrimiento", Harry irá haciendo otra serie de "descubrimientos" como los siguientes:

1 Todas las referencias al texto se harán a la traducción y adaptación que he hecho para Colombia de esta novela. Utilizo aquí la tercera edición de El descubrimiento de Harry, publicada en Bogotá en 2006; a esta edición corresponden los números de página citados. Las referencias al manual Investigación filosófica serán a la edición española de Ediciones de la Torre, de Madrid (Lipman, Sharp \& Oscanyan, 1988). 
- que lo que decimos puede ser expresado en un lenguaje que nos facilita el ejercicio de hacer deducciones correctas: el lenguaje de las proposiciones categóricas.

- que hay cuatro formas básicas de esas proposiciones categóricas:

- las proposiciones en A ("Todas las materias son interesantes").

- las proposiciones en E ("Ninguna materia es interesante").

- las proposiciones en I ("Algunas materias son interesantes").

- Las proposiciones en O ("Algunas materias no son interesantes").

- que, aunque la gran mayoría de las oraciones que usamos en nuestro lenguaje ordinario no empiezan por "todos" o "ningún", es posible reescribirlas en forma de oraciones que empiezan por "todos", "ningún" o "algunos" (con ello, no sólo descubre los tres cuantificadores básicos del lenguaje lógico, sino que empieza a comprender que es posible traducir de alguna forma las expresiones de nuestro lenguaje natural a la forma de las proposiciones lógicas).

- que es posible, entonces, encontrar (o "inventarse") un lenguaje en el cual podemos decir todo lo que queremos decir sin que caigamos en las ambigüedades, inexactitudes o confusiones de nuestro lenguaje ordinario.

A lo largo del texto irán apareciendo, además de los anteriores, otra serie de "descubrimientos" lógicos. Por ejemplo: el uso que se puede hacer de diagramas para examinar la validez de los razonamientos (pp. 24-27); las proposiciones que expresan ya no la inclusión o exclusión (parcial o total) de un individuo o una clase en otra clase, sino relaciones (como "más alto que...", "mayor que...", "igual a...") (pp. 56-58 y 62-67) y el hecho de que algunas de esas relaciones "se transmiten" de un par de frases a una tercera, es decir, son relaciones transitivas (pp. 64-67). Finalmente, sobre la base de esto, empieza a descubrir que hay inferencias deductivas que se pueden hacer ya no sobre la base de una premisa (como cuando de una sola proposición deducíamos otra), sino sobre la base de dos premisas, pues hay una relación que "se transmite" de una a otra proposición; tales deducciones, mediadas por un término que hace el enlace, son la base de los silogismos (cf. pp. 66-67 y 118-123). A ello se suman todavía más descubrimientos, como ciertas reglas básicas de inferencia lógica que descubre Toño en el capítulo dieciséis, como el modus ponens y el modus tollens.

Comprender cuál fue "el descubrimiento de Harry" implica comprender cómo se articulan, a partir de su primer descubrimiento, una serie de descubrimientos que se van sucediendo unos a otros y se van concatenando entre sí hasta llegar a conformar un lenguaje completo formado por ciertas formas estandarizadas de expresar nuestros pensamientos (las proposiciones categóricas) y una serie de reglas que rigen el modo como se puede de una proposición inferir válidamente otra. 
Lo que irá descubriendo Harry a medida que se desarrolla la historia es que, además, este "lenguaje", aunque sea artificial, resulta muy útil para varias cosas: (1) para pensar de forma más clara y ordenada, (2) para identificar más fácilmente errores de razonamiento (falacias) muy comunes en nuestros modos de expresión (como cuando descubre el error de razonamiento de doña Mercedes al final del capítulo primero) y, especialmente, (3) para descubrir que hay una serie de reglas que seguimos al desarrollar nuestros pensamientos y que, al hacer conscientes esas reglas, podemos descubrir de qué manera logramos razonar de una forma válida.

Para comprender mejor este "descubrimiento" de Harry es necesario hacer dos precisiones adicionales:

a. Lo que Harry está buscando desde el principio, y lo que va encontrando a medida que la historia se desarrolla, es un lenguaje en el cual puede expresar lo que piensa sin caer en las imprecisiones y ambigüedades del lenguaje ordinario: ese lenguaje es el de las proposiciones categóricas.

Ahora bien: ¿qué es una proposición categórica?

Es una forma de expresar nuestros pensamientos en la cual se hacen explícitas las relaciones lógicas que existen entre los términos de una oración que usamos.

Veámoslo en un ejemplo sencillo. Supongamos que alguien dice: Me gustan los Renault, porque son carros verdaderamente lindos". Los términos subrayados en la oración anterior en cierto sentido sobran porque no dicen nada acerca del sujeto de mi oración (los Renault), sólo indican un sentimiento de aprobación ("me gustan"), una conexión que hago en la frase para dar cuenta de ese sentimiento ("porque...") o un cierto énfasis para dar mayor fuerza a lo que estoy expresando ("verdaderamente"). Estos términos simplemente enfatizan y expresan o explican sentimientos. Sin embargo, en sentido estricto, carecen de valor lógico. La frase anterior se puede "reducir" a una expresión más sencilla donde se afirma lo mismo pero sin hacer énfasis o expresar emociones: Todos los Renault son carros lindos.

En esta última frase, que tiene la forma de una proposición categórica, se pueden ver los cuatro elementos básicos de una proposición: un cuantificador (todos), que indica la cantidad de los miembros de una clase a la que hacemos referencia; el sujeto (los Renault); un término que sirve de cópula, es decir, que une sujeto y predicado (y que en una proposición categórica es el verbo ser en alguna de sus formas es/son); y el predicado (carros lindos).

Expresada la oración de esta forma las relaciones lógicas existentes entre el sujeto y el predicado se hacen completamente explícitas: hay dos clases (la de las Renault y la de los carros lindos) y la primera de ellas está incluida totalmente en la segunda, es decir, todos los miembros de la clase de los Renault son a su vez miembros de la clase de los carros lindos. 
El descubrimiento de las cuatro formas básicas de las proposiciones categóricas (que se nombran con las vocales $\mathrm{A}, \mathrm{E}, \mathrm{I}, \mathrm{O}$ ) nos pueden servir para expresar una gran cantidad de pensamientos de una forma más clara y precisa. Este descubrimiento, que recorre todos los primeros cuatro capítulos de $\mathrm{DH}$, se sistematiza finalmente en la página 36.

b. Puesto que Harry está descubriendo o "inventando" un lenguaje, tiene que descubrir también unas reglas de traducción a ese lenguaje. Este es precisamente el problema que le plantea Toño al comienzo del segundo capítulo, cuando le pregunta cuántas de nuestras oraciones empiezan por "todos" o "ningún" (cf. p. 11). Harry descubrirá que, aunque efectivamente muy pocas de nuestras oraciones tienen esa forma, en principio todas nuestras oraciones podrían ser "traducidas" a esa forma. En esto recibe la ayuda del profesor Espinosa que, a lo largo del capítulo dos, y con ayuda de todos los miembros del curso, le ayuda a Harry a descubrir todas las formas posibles de decir "todos" en nuestro lenguaje ordinario. Con ello, éste logra darse cuenta de que muchas de las oraciones del lenguaje ordinario (tal vez, incluso, todas) se pueden "traducir" a proposiciones categóricas que empiezan por "todos" o "ningún".

Sin embargo, un nuevo problema se le plantea a Harry cuando, al comienzo del capítulo cuatro (p. 33), su mamá le pregunta cómo se diría en su lenguaje "en el cuarto hay siete sillas". Dado que Harry no encuentra una respuesta satisfactoria a la pregunta de su madre, la plantea nuevamente a sus compañeros en la clase del profesor Espinosa, y, a partir de una sugerencia de Miguel (pp. 33-36), se da cuenta de que, para referirnos a solo una parte de los miembros de una clase cualquiera, podemos utilizar el término "algunos". Con el descubrimiento de este tercer cuantificador (que nos sirve para construir proposiciones particulares afirmativas o negativas), Harry completa las cuatro formas básicas del lenguaje que está construyendo y empieza a tener más claras las "reglas de traducción" que andaba buscando.

La objeción de Toño al comienzo del capítulo dos tiene, además de lo anterior, un segundo elemento: el problema de la utilidad de su "descubrimiento". "¿De qué sirve saber eso?", pregunta Toño. Harry no lo sabe aún, aunque ya ha visto que podría ser útil a propósito de los desatinados comentarios de su vecina. En realidad el problema del valor práctico de la lógica es algo que Harry sólo irá clarificando a medida que se desarrolla toda la trama de la novela, y especialmente a propósito de la conversación que tendrá con Toño sobre la insistencia del padre de este último en que Toño "debe ser ingeniero", y fundamentalmente en la medida en que empiece a aplicar las reglas descubiertas, con ayuda de sus compañeros de curso, a problemas de orden ético, epistemológico, cosmológico, estético, político, educativo, etc. en los capítulos posteriores (especialmente del capítulo cinco en adelante). 
Recojamos ahora todo lo anterior e intentemos decir en unos pocos ítems qué fue lo que descubrió Harry. Lo que descubrió Harry, dicho brevemente, fue lo siguiente:

1. Que lo que decimos y pensamos puede ser expresado en un lenguaje más claro y preciso que el lenguaje ordinario: el de las proposiciones lógicas.

2. La estructura general y las cuatro formas básicas de las proposiciones categóricas.

3. Que hay unas "reglas de traducción" que permiten "pasar" oraciones del lenguaje ordinario al lenguaje de las proposiciones lógicas.

4. Que este "lenguaje del pensamiento" (o estas "reglas del pensar", como él las llama) pueden ser útiles para pensar con mayor claridad y para razonar con orden.

A estos descubrimientos básicos (que, en lo esencial, están ya desarrollados en los primeros cuatro capítulos de la novela) se articularán posteriormente, en los capítulos sucesivos, nuevos "descubrimientos", entre los cuales podemos destacar los siguientes:

- Los límites que tienen las formas inductivas de razonamiento, pues se apoyan en una tendencia natural de nuestra mente a "sacar conclusiones precipitadas" (cf. pp. 38-39).

- La forma de las proposiciones que expresan relaciones y el hecho de que algunas de esas relaciones son "transitivas" y otras no (caps. 7 y 8).

- La forma del razonamiento silogístico (especialmente en el cap. 14).

- Las formas de inferencia inmediata, como la conversión, la obversión y la contraposición (especialmente a partir del capítulo 12).

- Las posibles relaciones de oposición que pueden existir entre las proposiciones lógicas, y que son básicamente tres: la contrariedad, la subalternación y la contradicción.

- Algunas reglas de inferencia lógica, como la del silogismo hipotético (cf. p. 69), o el modus ponens y el modus tollens (cf. cap. 16).

La comprensión del sentido y función de cada uno de estos "descubrimientos" sólo es posible verla con mayor claridad si, a su vez, intentamos comprender la estructura de una obra como DH. Puesto que ésta es una novela que tiene una intención filosófica explícita, es una "conjugación" de dos estructuras distintas: una teórica y otra narrativa. Ocupémonos más despacio de este punto. 


\section{La estructura (teórica y narrativa) de DH}

\subsection{Las dificultades propias de una novela sobre lógica formal}

Como lo señalamos ya al comienzo de estos "apuntes", cada novela del programa FpN es un programa (el propio Matthew Lipman, cuando se refiere a estos textos no suele hablar tanto de "novelas" como de "programas"; así, por ejemplo, en muchas de las referencias que hace al texto que estamos introduciendo habla de "Harry program"). Pues bien, como decíamos, DH es un programa de lógica formal.

Lo anterior en buena parte explica la aridez de cierta parte del texto y el hecho de que muchas veces a los propios niños y jóvenes para los que fue escrito les parezca por momentos "distante", "abstracto", "extraño". No siempre es fácil que un muchacho de doce años se identifique con Harry, pues, quienes leen esta historia no siempre se sienten atraídos por los problemas "abstractos" que aquí se plantea el protagonista. No se sigue de allí, por supuesto, que esto justifique el que el texto no se trabaje con los muchachos de estas edades. Esto indica, más bien, que un texto como DH planteará a los niños y jóvenes "retos cognitivos" más altos que la simple lectura de una historia de aventuras o de cualquier "best seller" que pueda encontrarse en el mercado.

La lógica formal -todos lo sabemos- no es asunto de fácil comprensión, a la vez que no es un tema que resulta ordinariamente muy motivador para quien se enfrenta con él por primera vez. Es algo árido, difícil, e incluso, la mayoría de las veces, puede resultar aburridor, pues a menudo su enseñanza se reduce a la memorización y aplicación mecánica de reglas de razonamiento, muchas de las cuales, además, parecen simplemente triviales. Aunque en DH todas estas "reglas" se presentan en un contexto dramático en donde se van descubriendo paso a paso y ligadas a asuntos filosóficos que pueden interesar a cualquier persona (preguntas sobre la mente, los sueños, la forma correcta de actuar en determinadas situaciones, etc.), la verdad es que a menudo los aspectos centrales -los estrictamente lógicos- de DH suelen pasar inadvertidos no sólo para los niños y jóvenes que leen y discuten la novela, sino incluso para los profesores que trabajan con ella.

Podríamos decir que, en general, no es fácil "ver" toda la "trama lógica" de DH para quien no tiene por lo menos algunos conocimientos básicos de lógica formal. No suele ser fácil ni siquiera para quienes han recibido una cierta formación sistemática en esta materia. Lo he podido comprobar, por ejemplo, con un buen número de alumnos universitarios de una clase de Lógica en la Carrera de Filosofía, a quienes les propongo la lectura de DH después de haber estado trabajando sistemáticamente la lógica formal a lo largo de por lo menos dos meses (con cinco horas semanales de clase y varias horas de trabajo práctico resolviendo ejercicios de razonamiento lógico). Muchos de ellos, cuando les 
pido que me expliquen alguno de los problemas básicos estudiados en clase a partir de la trama narrativa de $\mathrm{DH}$, se ven cortos a la hora de descubrir en el relato los problemas que hemos venido trabajando sistemáticamente durante los dos meses anteriores. Debo decir que, incluso para mí mismo, no ha sido fácil percibir el modo como los "descubrimientos" de Harry se van articulando sistemáticamente hasta constituir un programa básico de lógica deductiva. La estructura lógica del texto sólo he logrado clarificarla -y aún con algunos vacíos- después de algunos años de estudiar con cierta sistematicidad la lógica formal en los textos clásicos que existen para tal fin, como las obras de Irving Coppi y Cohen/Nagel. Por supuesto, para quien no tiene una formación filosófica básica (ni una formación específica en lógica formal) el asunto debe ser todavía más complicado.

De lo anterior no debe inferirse, desde luego, que la comprensión de DH esté reservada "para filósofos". Lo que quiero poner de presente, más bien, es que la comprensión de esta obra supone tanto una atención muy detenida a la hora de leer la novela (para ello, sin duda, el manual para el profesor que la acompaña, que tiene por título Investigación filosófica, es una gran ayuda) como un cierto esfuerzo personal de estudio de algunos de los fundamentos básicos de la lógica formal².

Quiero insistir en este punto: si no se comprende la "trama lógica" de DH, este texto no pasará de ser una historieta artificial en donde un grupo de niños juegan con frases (por ejemplo, a invertirlas, o a reducirlas en su contenido). A quienes no ven en esta obra más que una historieta juvenil les resulta, además, muy sospechoso el hecho de que muchachos de alrededor de 12 años (casi niños todavía) puedan plantearse los problemas que allí se plantean y descubrir lo que allí descubren. Sin embargo, los muchachos de esta edad están en capacidad de razonar de una forma tan ordenada y coherente como lo puede hacer un adulto, y a menudo, si no lo hacen, es porque no se encuentran suficientemente motivados para ello, o porque los propios adultos no se los exigimos o no se los permitimos. He trabajado también este texto con niños de séptimo grado (que tienen, en promedio, entre 11 y 12 años) y he utilizado, para introducirlos en el razonamiento lógico, el mismo tipo de enigmas que suelo usar con los alumnos universitarios de filosofía, obteniendo no sólo resultados semejantes, sino, a veces, incluso mejores, pues tienen una capacidad intuitiva que a menudo en los adultos se encuentra ya oscurecida.

DH no es, entonces, a pesar de todo lo dicho hasta aquí, un texto inaccesible para los no-filósofos. Ni siquiera lo es para los niños (conozco casos de niños de educación preescolar que se han interesado por discutir, y que efectivamente han discutido entre sí, algunos pasajes de esta obra, incluso algunos de los más "abstractos", como las discusiones en torno al significado de la mente que hay

2 Para empezar, podría ser suficiente con la lectura de los capítulos 5, 6 y 7 de la Introducción a la lógica de Irving Coppi y Carl Cohen (2007). Ello se podría complementar también con la lectura de los capítulos 2, 3 y 4 de la Introducción a la lógica y al método científico de Morris Cohen y Ernest Nagel (1990). Son textos que se deben leer despacio, tomando atenta nota y realizando los ejercicios de razonamiento que allí se proponen. 
en los capítulos tres y seis). El propio autor, Matthew Lipman, experimentó su texto trabajando con niños de quinto grado en el año 1970, obteniendo resultados bastante alentadores.

\subsection{Estructura teórica y estructura narrativa}

Ahora bien. Una adecuada comprensión de DH supone una comprensión específica de su estructura teórica. Para ello, como ya decíamos, es muy importante el estudio del manual Investigación filosófica. Sin embargo, ello puede ser también insuficiente, pues éste es sobre todo un material práctico; además, por desgracia, éste es precisamente el único manual para el cual Lipman no escribió una introducción teórica; y es, tal vez, el que más lo requería. Por supuesto, una adecuada comprensión de la estructura teórica supone, a su vez, un cierto grado de familiaridad con la estructura narrativa de la novela, asunto que sólo se logra con una lectura atenta y reflexiva del texto (no basta, por supuesto, con la primera lectura, puramente informativa, sino que se requiere de varias lecturas sucesivas en las que se va ahondando en su problemática).

Para empezar, lo esencial en esta serie de lecturas sucesivas es que nos familiaricemos con los personajes de la novela (y con los diversos "estilos cognitivos" que cada uno de estos personajes representa), con el modo como las situaciones se van creando y resolviendo a lo largo del relato, con la forma de las descripciones y los diálogos, y con la manera como los descubrimientos que van haciendo Harry y sus compañeros de curso se logran sistematizar y formalizar con la ayuda de los adultos que interactúan con ellos en la novela (especialmente personajes como el profesor Espinosa, la señorita Myriam Jaimes o los propios papás de Harry).

He distinguido en el párrafo anterior entre la estructura teórica y la estructura narrativa de $\mathrm{DH}$. Con el término "estructura teórica" me refiero básicamente a toda la armazón conceptual que da significado y orden a la trama de los acontecimientos que se suceden en la novela; esta "trama de acontecimientos" y el modo como se relacionan unos con otros es precisamente lo que llamo aquí "estructura narrativa". Las partes de una estructura teórica son conceptos, los de una estructura narrativa son acontecimientos relatados. Entonces, una buena forma de tener un primer acercamiento a la estructura teórica de DH podría ser, dando una mirada al índice del manual que acompaña a la novela, en donde se puede observar el modo como las ideas principales que guían el desarrollo del relato van apareciendo secuencialmente y se van articulando unas con otras. La mejor forma de percibir la estructura narrativa del texto es mediante una lectura atenta de él (ojalá una lectura "de corrido" de toda la novela).

Desde luego la estructura teórica y la estructura narrativa de $\mathrm{DH}$ no son dos cosas separadas, sino, por el contrario, dos elementos que, aunque distintos, se encuentran perfectamente articulados. Al señalar estas dos "estructuras", el 
interés está en distinguir (para comprender mejor), no en separar lo que, de hecho, constituye una unidad.

He de decir, sin embargo, que en DH la estructura narrativa depende enteramente de la estructura teórica, y la primera sólo es comprensible a la luz de la segunda. Existe una supremacía de la estructura teórica sobre la estructura narrativa. Esto, por cierto, es lo propio de una "novela filosófica", en donde lo esencial es el modo como se articulan los conceptos, y en la cual los personajes (si bien poseen cierta autonomía, como sucede en la construcción de cualquier relato) representan conceptos, o responden a determinados estilos cognitivos que el escritor quiere poner de presente en la construcción de su trama.

En esto una novela o un cuento filosófico difiere radicalmente de cualquier otro tipo de historia: en que es sobre la base de una estructura conceptual, o incluso de la exploración de un problema filosófico, que se van definiendo y caracterizando los personajes (que se determinan sus nombres, sus cualidades, sus rasgos cognitivos y emocionales) y que se va definiendo la trama narrativa, que implica tanto descripciones de los ambientes en que se desarrollan los acontecimientos como descripciones de lugares, de personas, de gestos, de actos mentales. Es también a la luz de la estructura teórica que, en un relato filosófico, se construyen los diálogos o los monólogos reflexivos de los personajes. Para ilustrar esta tesis presentaré dos testimonios sobre ello.

El primero es propio. En el último tiempo he empezado a escribir algunos cuentos y novelas filosóficas para niños. Tras muchos años de dedicarme a traducir y adaptar los textos escritos por Lipman, me he decidido, por fin, a intentar escribir textos propios. Hace un tiempo escribí una novela para niños entre cinco y seis años que tiene por título Checho y Cami. Tenía muchas ideas en la cabeza y recordaba un sinnúmero de escenas en las que distintos niños (mis propios hijos y muchos otros) se preguntaban cosas, tenían ocurrencias, hacían comentarios ilustrativos de sus procesos mentales. Para poder vincular todos estos elementos $\mathrm{y}$, a partir de ello, construir un relato que tuviera tanto una consistencia narrativa como una estructura a la vez ágil y coherente, tuve que diseñar primero, en un papel, una especie de "mapa conceptual" que me guió todo el tiempo a medida que escribía el relato. Ese "mapa conceptual", en donde aparecían ubicados los distintos conceptos con que trabajaría y podían verse las conexiones existentes entre ellos, me mostraba toda la estructura de lo que quería escribir. Así mismo, ese "mapa conceptual" me permitía establecer cada vez nuevas conexiones, al tiempo que establecía los límites precisos dentro de los cuales se movería mi narración.

Una vez tuve clara esta estructura teórica de mi relato los personajes se fueron igualmente delineando en mi mente. Así me fue posible empezar a caracterizarlos y ponerlos a dialogar entre sí y conmigo mismo. A medida que empecé a escribir la historia fue necesario hacer nuevas precisiones sobre el modo como procedería (empecé a entender, por ejemplo, que la historia debía ser narrada "a dos voces" por un niño y una niña y que, cada cierto tiempo, deberían aparecer 
distintos modelos de diálogo -entre niños y entre niños y adultos-). Una vez se fueron definiendo todos estos elementos, e incluso pude fijar un límite a la extensión del relato, puedo decir que la historia prácticamente "se escribió sola". Aunque los personajes seguían mis orientaciones (y yo, a la vez, me dejaba guiar por el mapa conceptual que había construido), éstos adquirieron vida propia y fueron, paso a paso, contándome su historia. El trabajo que viene después de escribir un relato de éstos es el de intentar describir con un inmenso rigor analítico esa trama teórica, filosófica, que le da sustento y vida al relato que podrán leer el día de mañana los potenciales lectores de ese texto.

El segundo testimonio es de Matthew Lipman que se refiere específicamente al modo como surgió DH y, con esta novela, al conjunto del programa FpN. Sus palabras al respecto son las siguientes:

¿Era posible ayudar a niñas y niños a pensar más diestramente? No tenía dudas de que niñas y niños pensaban con la misma naturalidad con la que hablaban o respiraban. Pero, ¿cómo lograr que pensaran bien?

Me sugirieron la idea de un cuento para niños. Pero, ¿qué tipo de cuento? Seguro que no aquél en que los adultos que todo lo saben explican benévolamente al pequeño ignorante las diferencias entre pensar bien y pensar mal. Nada tan condescendiente. Debería ser algo que los jóvenes lectores pudieran descubrir por sí mismos, con poca ayuda de los adultos. Los niños de la historia deberían constituir, de alguna manera, una pequeña comunidad de investigación, en la que cada cual participara, en alguna medida, de la búsqueda cooperativa y del descubrimiento de modos de pensar más efectivos. Mi idea era que el grupito de niños de la historia sirviera de modelo con el cual los niños y niñas reales de la clase pudieran identificarse. Una imagen semejante, de niños viviendo juntos de manera inteligente y respetándose mutuamente, podría dar a niñas y niños la esperanza de que semejante ideal era factible (como habían hecho los diálogos de Platón con los adultos).

Puedo recordar cuando deliberaba acerca de la tarea preliminar de nombrar a los personajes de la historia (...). Se me ocurrió la idea de jugar con el nombre del fundador de la lógica (Aristóteles). ¿Qué tal Harry Startle? Demasiado oscuro y rebuscado. Me decidí por Harry Stottlemeier. Los nombres de los otros personajes me fueron sugeridos por diferentes líneas étnicas, una maniobra conscientemente adoptada por Moby Dick (de Herman Melville).

Los personajes no los tomé de la vida real, así como tampoco la trama. Mis propios hijos tenían ocho y nueve años de edad en aquel momento, pero no pensé en usarlos como modelos. Ellos me dieron, fundamentalmente, el sentido de las posibilidades de diálogo con las personas jóvenes. 
Los niños (de la historia) se diferenciarían unos de otros, me parecía, no tanto por sus modos de ser cuanto por sus estilos de pensamiento. Uno sería empirista, otro intuitivo, un tercero analítico, un cuarto escéptico, etc., aunque ningún estilo se presentaría como inherentemente mejor o peor que otro. Harry debería ser paciente y explorador, alternando entre el maravillarse, el preguntarse y el dudar sobre sí mismo. Tenía la sospecha de que sus habituales confusiones y las situaciones embarazosas en que se encuentra lo acercarían a niñas y niños más fácilmente de lo que lo harían las virtudes de un protagonista menos falible.

En cuanto a la trama de la historia, la pensé como un paradigma de investigación en miniatura. Los niños descubren una regla lógica, la conversión (encontrar la oración conversa). La prueban en una cantidad de oraciones diferentes y funciona. Pero alguien encuentra un contraejemplo. En un primer momento aparece la consternación y la desesperanza. Sin embargo, luego se dan cuenta de que el principio puede ser extendido hasta abarcar el contraejemplo. Una vez más aparece la prueba; cuando se la revisa, la regla aparece como válida. Pero, ¿funcionará en la vida real? El episodio final del capítulo brinda a niñas y niños una oportunidad de aplicar su descubrimiento en una situación extraescolar: ¡y funciona!

Estas fueron algunas de las circunstancias inmediatas relativas a la redacción del primer capítulo de El descubrimiento de Harry. Pero, en ese momento, llegué a la conclusión de que lo que necesitaba no era un episodio, sino una novela. Para que pudiera persuadir, el tema del descubrimiento debería ser ampliado y reforzado. Y los principios del razonamiento descubiertos por niñas y niños deberían aparecer como relevantes para los aspectos más fundamentales y problemáticos de sus vidas (Lipman, 1992, pp. 3-7)3.

En estos dos casos que acabo de presentar resulta evidente de qué manera la estructura teórica de un texto, guiada por una intención específicamente filosófica, es lo que determina su estructura narrativa. Ello no implica, desde luego, que la trama narrativa sea secundaria o puramente accidental, sino, más bien, que debe ser estrictamente coherente con la coherencia teórica de un texto como éste.

\subsection{Un primer esbozo de la estructura teórica de DH}

Tal vez esta relación entre la estructura teórica y la estructura narrativa de un relato filosófico se logre clarificar un poco más si describimos por aparte cada una de ellas. Intentaré a continuación hacer un primer "esbozo" de lo que considero esencial en la estructura teórica de DH. Se trata sólo de un esbozo que, por

3 Existe una traducción al español, de la argentina Vera Waksman, de este texto, publicada en Kohan y Waksmann, 1997, pp. 153-158. La parte citada es de las pp. 154-155. La lectura de este texto resulta muy iluminadora no sólo sobre el origen de $\mathrm{DH}$ sino, en general, de todo el programa FpN. 
supuesto, acepta todos los retoques que se consideren necesarios. Para intentar describir esa estructura, nada hay mejor que intentar identificar sus "partes", aclarando que cuando utilizo este término en ningún caso debemos entender tales "partes" como cosas separadas, sino como elementos que conforman una estructura y que mantienen entre sí una interacción dinámica.

Mi lectura de DH me ha permitido identificar en el texto por lo menos cuatro partes básicas:

1. La construcción del "lenguaje" de la lógica formal (que abarca esencialmente los cuatro primeros capítulos de la novela) y que llamaría "la primera fase de descubrimiento". Allí, a partir del primer descubrimiento (el de la no-convertibilidad de las proposiciones en A), se van dando otra serie de "descubrimientos" como los siguientes:

- La estructura y forma de las proposiciones categóricas.

- Algunas reglas de traducción del lenguaje ordinario al lenguaje de la lógica formal.

- La posible utilidad de la lógica formal.

- Una primera exploración en torno al significado de la mente.

- Una forma sencilla de diagramación para probar la validez de razonamientos.

- La búsqueda de un conjunto de "reglas" que rigen nuestros procesos de pensamiento correcto.

- Una primera exploración de la conexión existente entre lógica e imaginación (con ocasión del sueño que tiene Elisa en las páginas 23-24 del texto).

2. Un interludio filosófico (que abarca esencialmente los capítulos 5 a 11), en donde se da la ocasión para aplicar los primeros descubrimientos lógicos a distintos problemas teóricos y prácticos de la vida de los jóvenes. Sobre la base del lenguaje lógico adquirido, en estos capítulos Harry y sus amigos van abordando el examen de distintos problemas filosóficos, entre los cuales podemos destacar los siguientes:

- El sentido que tiene la enseñanza y el aprendizaje escolar (cap. 5).

- El significado de los "actos mentales" (como "soñar" o "tener una idea").

- La relación existente entre pensamiento y lenguaje.

- El carácter cultural del ser humano.

- La resolución de dilemas éticos y el examen de la argumentación presente en ellos, especialmente a propósito de la situación planteada por Daniel cuando no quiso ponerse de pie para saludar a la bandera (caps. 9 y 10). 
El planteamiento de estos problemas filosóficos va unido aquí a dos asuntos adicionales:

- Una exploración de las "mentes" de los personajes (cf. caps. 8 y 11) que ayuda a los niños y jóvenes que leen la novela a intentar entrar en las mentes de otros, y que va definiendo de una forma cada vez más clara los perfiles cognitivos de los personajes.

- Nuevos "descubrimientos" lógicos que, aunque por ahora son independientes, se empezarán a conectar y estructurar a partir del capítulo 12, y entre los cuales destacamos los siguientes:

- Los límites de la inducción y los peligros de una generalización precipitada (cap. 5).

- Las "diferencias de grado" y las "diferencias de especie" (cf. pp. 53-54).

- Las proposiciones que expresan relaciones (cap. 8) y el hecho de que algunas de esas relaciones son transitivas y otras no.

- La transitividad como base para la elaboración de inferencias mediatas, lo que dará lugar luego al descubrimiento de la forma del silogismo.

- La lógica propia del razonamiento moral (caps. 9 y 10).

3. El descubrimiento de la estructura y reglas de un argumento simple (que abarca fundamentalmente los capítulos 12 a 16) y que constituye lo que podríamos llamar "la segunda fase de descubrimiento". El capítulo 12 es muy importante como un "punto de ruptura" de la trama de DH. Dicho capítulo comienza con una conversación entre Harry y Elisa en la cual éste le dice a su amiga: "hace semanas que no conseguimos nada" (p. 92). Esto es cierto sólo parcialmente, pues ya han descubierto en los capítulos previos algo esencial: que hay relaciones "que pasan" (relaciones transitivas).

Veámoslo en el siguiente ejemplo:

Si yo sé que Juan es mayor que Pedro, y que Pedro es mayor que Gustavo, puedo, si examino bien estas dos proposiciones, deducir una tercera. Así:

Si Juan es mayor que Pedro.

Y si Pedro es mayor que Gustavo.

Entonces, necesariamente Juan es mayor que Gustavo.

Este razonamiento, tan elemental como habitual, nos permite descubrir dos cosas, que son las que han venido descubriendo Harry, Elisa y sus demás compañeros: 
a. que hay relaciones que son transitivas, es decir, que pueden "pasar" de dos proposiciones a una tercera, lo que nos permite construir algunas formas de argumento simples pero muy útiles, como el silogismo hipotético del ejemplo anterior.

b. que, por lo tanto, deben existir algunas reglas que nos permitan extraer de dos o más proposiciones dadas nuevas proposiciones; es decir, que hay reglas deductivas que nos permiten construir argumentos correctos.

A esta altura de su reflexión (comienzos del cap. 12), Harry y Elisa aún no se han dado cuenta de las implicaciones que tienen los descubrimientos que han venido haciendo en los últimos capítulos, y por eso deciden volver a lo único que tienen claro: las cuatro formas básicas de las proposiciones categóricas (cf. pp. 92 y ss).

Sin embargo, como ya insinuábamos, aquí empieza una segunda fase de descubrimiento, que tiene que ver ya no tanto con el descubrimiento de un lenguaje lógico cuanto con la forma de proceder para construir argumentos correctos. A medida que, a partir de la observación atenta de las cuatro formas básicas de las proposiciones lógicas (pp. 92 y ss), se dedican a reflexionar sobre ellas, Harry y Elisa (y luego algunos otros compañeros de ellos) van descubriendo otra serie de cosas, que siguen una cierta secuencia. Descubrimientos como estos:

- Las relaciones de oposición entre proposiciones, que son básicamente tres:

${ }^{*}$ ) Contrariedad (dos proposiciones son contrarias cuando, aunque las dos no pueden ser verdaderas, las dos pueden ser falsas; así las proposiciones en A ("todas las materias son interesantes") y en E ("ninguna materia es interesante") son contrarias, pues no pueden ser las dos verdaderas, pero podrían ser las dos falsas, dado que es posible que lo verdadero es que algunas materias sean interesantes y otras no lo sean.

(*) Subalternación (dos proposiciones son subalternas cuando la verdad de una implica la verdad de la otra. Las proposiciones en I ("algunas materias son interesantes") son subalternas de las proposiciones en A ("todas las materias son interesantes"), dado que, si es cierto que todas las materias son interesantes, es necesariamente cierto que algunas lo son.

(*) Contradicción (dos proposiciones son contradictorias cuando la verdad de una implica necesariamente la falsedad de la otra. De esta forma, las proposiciones en A ("todas las materias son interesantes") y en O ("algunas materias no son interesantes") son necesariamente contradictorias, pues, si una de ellas es verdadera, la otra necesariamente sera falsa, pues basta, por ejemplo, con afirmar que es verdadero que hay algunas materias que no son interesantes para que no podamos afirmar que todas lo son.

El conocimiento de estas reglas elementales (que se refieren a las relaciones de oposición entre proposiciones) es esencial, pues nos ofrece bases para hacer una 
serie de inferencias inmediatas, es decir, una serie de deducciones en torno a la posible verdad o falsedad de determinadas proposiciones.

- El análisis de posibilidades, que les permite a Harry y sus amigos examinar un problema de orden cosmológico en torno al origen del mundo (cap. 13, pp. 101 y ss).

- La estructura del silogismo y sus reglas de validez, así como técnicas para determinar la validez de un razonamiento silogístico como la llamada "analogía lógica" (cf., en pp. 122-123, el modo como Elisa y María Fernanda examinan el razonamiento que acaban de escuchar). (Todo esto especialmente en el capítulo 14).

- Algunas reglas generales de inferencia lógica, como el modus ponens y el modus tollens, en el capítulo 16.

En general, podríamos decir que toda esta tercera parte de DH está entrelazada y es prácticamente simultánea con una cuarta parte, en la cual estos descubrimientos que van haciendo los personajes de la novela se aplican a problemas filosóficos de diversa índole.

4. Aplicación de los "descubrimientos" lógicos a problemas de filosofía teórica y práctica (que corresponde aproximadamente a los capítulos 13-17). En los últimos capítulos de DH se entrelazan el descubrimiento de la estructura y reglas de un argumento simple (hasta llegar al descubrimiento de la forma del silogismo) con el examen de otros problemas filosóficos "de contenido"4 como los siguientes:

- Problemas cosmológicos, como el caso de si el mundo es eterno o tuvo un comienzo (en el capítulo 13), para cuyo examen se utiliza la estrategia de análisis de "las cuatro posibilidades" propuesta por Toño).

- Problemas estéticos de diverso orden, como los que se plantean con ocasión de la visita de Susy y Ana María al museo de arte (cap. 14).

- Problemas políticos como los que se refieren a los derechos ciudadanos (planteados por María Fernanda en el capítulo 14) o la discusión sobre la guerra que tiene Harry con su papá (cap. 15, pp. 125-127).

- Problemas epistemológicos, como la distinción entre describir y explicar, a propósito de la explicación del profesor Barrios sobre "los vasos comunicantes" (cap. 15, pp. 127-131).

- Asuntos como la distinción entre pensamiento analítico y pensamiento intuitivo, representados en las figuras de Toño y Elisa (caps. 16 y 17).

4 Algunos problemas de filosofía de la educación, filosofía de la mente, filosofía del lenguaje, antropología filosófica, ética normativa y metaética habían sido ya examinados en la segunda parte (cap. 5-11). 
Esta descripción, necesariamente genérica y excesivamente analítica -a tal punto que, aunque permite ver las partes, no permite ver muy bien las conexiones orgánicas entre estas partes-, de la estructura teórica de DH se ha hecho, sin duda, en clara conexión con la trama de acontecimientos de la novela. Es decir, en relación explícita con la estructura narrativa del texto. Debemos ahora mirar, para completar nuestra comprensión sobre la estructura general del texto algunos aspectos particularmente importantes de eso que hemos llamado su estructura narrativa.

\subsection{Algunos elementos a tener en cuenta de la estructura narrativa de DH}

¿Qué podemos decir de la estructura narrativa de un texto como éste? No mucho en principio. Por lo menos, no mucho más que lo que cualquier lector atento puede descubrir con una lectura juiciosa del texto ${ }^{5}$. Sin embargo, quisiera insistir ahora en "la otra cara de la moneda". Si bien es cierto que he afirmado que, por la intención específicamente filosófica del texto, prima en él su estructura teórica por sobre su estructura narrativa, ello no quiere decir, de ninguna manera, que la estructura narrativa de DH sea completamente secundaria o puramente accidental. Hay aquí rasgos específicos que no debemos dejar pasar por alto. Me limitaré por lo pronto solamente a destacar algunas estrategias narrativas que es importante tener en cuenta a la hora de acercarse a un texto como éste.

Destacaré aquí, entonces, seis estrategias narrativas básicas:

1. La presencia de un narrador externo: Aunque, según lo confesó alguna vez Lipman, no es esto lo más deseable (él mismo dijo alguna vez que, si volviera a redactar DH, el narrador sería el propio protagonista, Harry, como ocurre en las novelas que ha escrito para los niños de la escuela primaria), creo que la estrategia de un narrador externo tiene algunas ventajas que no conviene soslayar:

- Permite una descripción más "objetiva" de los espacios, ambientes, gestos y sentimientos de los personajes (especialmente del protagonista), etc.

- Esto le da un tono menos "subjetivo" a la novela, algo que considero importante en un texto que está hecho para muchachos que están empezando a entrar en la adolescencia, que quieren empezar a ser adultos y que, puesto que tienen una perspectiva menos egocéntrica que la de los niños pequeños, buscan un descentramiento de su propia perspectiva que les permita pensar de una forma más autónoma.

5 Por supuesto no hay nada mejor para la comprensión de esta estructura narrativa que una lectura completa y reflexiva del texto (ojalá "de una sola sentada"); y nada mejor puede hacer el lector de estos "apuntes", que hacer, incluso antes de leer estas notas, una lectura personal de la novela. 
- Es ésta una estrategia necesaria (si se compara DH con las novelas para los más pequeños) en la medida en que abre la posibilidad de trabajar con un mayor número de personajes (los compañeros de Harry, que son más de 20, así como sus maestros y sus respectivas familias). Puesto que, en esta novela y en las que le siguen, la trama de personajes es muy compleja, la existencia de un narrador externo que nos va llevando de un ambiente a otro (de una familia a otra, del colegio a la familia, al grupo de amigos) es mucho mejor, pues permite una facilidad de desplazamiento mucho mayor a la narración.

- La ventaja principal, en mi concepto, es que el protagonismo en este caso se desplaza de un personaje (Harry) hacia un acontecimiento básico: el pensamiento y las reglas que seguimos para pensar de un modo correcto, a la vez que el proceso de búsqueda y descubrimiento de ello. El protagonista esencial de DH no es un personaje, sino el acontecimiento del pensar.

2. La configuración de los personajes: la amplia gama de personajes existentes en $\mathrm{DH}$ tiene una función central en la trama de la novela, pues pretende presentar distintos estilos y modelos cognitivos. Tales estilos y modelos se pueden ver tanto en los muchachos que aquí participan como en los adultos que acompañan sus procesos de indagación (padres y maestros). Aunque aquí sólo los enunciaremos, sin entrar a profundizar en ello $^{6}$, podríamos decir que, entre los muchachos de la historia, podríamos hacer las siguientes caracterizaciones generales: Toño representa ante todo una forma de pensamiento analítico, mientras que Elisa es fundamentalmente intuitiva, Marcos es el modelo más claro de pensamiento crítico, Harry representa la forma más explícita de pensamiento reflexivo, Ana María y Susy representan formas distintas de pensamiento creativo y María Fernanda una forma de pensamiento solidario ${ }^{7}$. En el caso de los padres y maestros podemos ver también estilos y modelos cognitivos bien diferenciados: los hay con ciertos visos autoritarios (como el profesor Pardo y, en alguna medida, el papá de Toño), los hay "exigentes", pero abiertos (como la señorita Jaimes o el papá de Harry), los hay abiertos a un diálogo democrático (como el profesor Espinosa o los papás de Harry). Cabe observar, sin embargo, cómo a lo largo del relato, cada uno de estos personajes se va modificando en la medida en que entra en diálogo y aprende a ponerse en la perspectiva de otros. El diálogo investigativo en el ámbito de una comunidad es la clave de dicha "transformación".

3. La interacción de tres contextos vitales (escuela, familia, grupo de amigos): Aunque el ambiente esencial en torno al cual gira toda la trama narrativa de DH es el colegio (como ocurre, igualmente, en todas las novelas del programa $\mathrm{FpN}$ ), este ambiente va, poco a poco (ya desde el tercer capítulo de DH), "desplazándose" hacia otros dos ambientes esenciales en la construcción de 
la identidad personal de un preadolescente o adolescente: su propia familia y su grupo de amigos. Hay también distintos tipos de familia: algunas "en crisis" (como la de Juanita), otras destrozadas por una tragedia (como el asesinato del padre de Guillermo), otras que representan modelos familiares propios de minorías étnicas (como la de Lucho); y también otras, como la de Harry, donde hay un ambiente permanente de diálogo y cooperación. Los grupos de amigos también presentan diversas formas de interacción (conviene ver al respecto, por ejemplo, a quiénes invita en cada caso Harry a quedarse en su casa, o los distintos grupos de niñas que asisten a un museo o una película). En realidad, esos "grupos de amigos" representan cierta comunidad de intereses entre ciertos personajes. De todas formas, de estos tres ambientes o contextos vitales de interacción prima el ambiente escolar, por ser el lugar fundamental de socialización hoy para un adolescente.

4. El paso del "descubrimiento" a la formalización de lo descubierto: los diversos "descubrimientos" que van haciendo Harry y sus compañeros (especialmente las "reglas del pensamiento" que van descubriendo a medida que se desarrolla la trama narrativa de la novela) no servirían de nada, o al menos de muy poco, si tales "descubrimientos" no se fueran organizando y sistematizando. Por ejemplo, Harry descubre que las oraciones que empiezan por "todos" no se pueden invertir porque dejan de ser verdaderas (y luego, con Elisa, descubren que no ocurre lo mismo con las oraciones que empiezan por "ningún"). Esto no es mayor cosa. Pero, cuando, ante el problema que le plantea Toño, se pone a buscar términos que sustituyen a "todos" en el lenguaje ordinario, entonces empiezan a organizarse los descubrimientos que vienen haciendo, hasta que, al final del capítulo cuatro, esto se sistematiza en una estructura básica: la de las cuatro formas de las proposiciones categóricas. Lo que Harry ha descubierto puede ser "transferido" a nuevas situaciones porque han logrado encontrar una estructura en donde lo descubierto aparece organizado estructuralmente según reglas que se pueden describir y explicar (lo que empezará a hacer a partir del capítulo doce). En este "paso" del descubrimiento a la formalización de ello, que organiza y consolida lo aprendido, que lo hace flexible y práctico, es, sin duda, esencial la participación del adulto. Aunque muchas veces son los papás (el de Toño, el de Harry y otros) los que ayudan en esto, en DH la figura central, en este aspecto, es el profesor Espinosa: es él el que les ayuda a identificar los términos del lenguaje ordinario que equivalen a "todos" (cap. 2), el que les ayuda a identificar las proposiciones categóricas (cap. 4), el que les ayuda a reducir a reglas de inferencia lo que han descubierto a propósito de la llegada tarde al colegio por parte de Toño (cap. 16). En todos estos momentos de "síntesis", que son momentos de organización y formalización de lo aprendido, está casi siempre el profesor Espinosa.

Estos momentos, que ordinariamente requieren la presencia de una mente adulta que es capaz de operar lógicamente (como la de este profesor), son esenciales en un proceso de aprendizaje cooperativo como el que se da en $\mathrm{DH}$, pues son los que garantizan que se organicen en estructuras las reglas descubiertas; y sólo esto hace posible una efectiva transferencia del aprendizaje. 
5. El estrecho vínculo entre lógica e imaginación: $\mathrm{DH}$ es una historia imaginaria (el mismo Lipman dice que no tomó sus personajes "de la vida real") sobre un tema tradicionalmente árido y difícil: las reglas y principios de la lógica formal. El supuesto básico de este texto es que, si uno puede descubrir las reglas de la lógica formal en un ambiente significativo (aunque éste sea ideal), podrá comprenderlas mejor y encontrarles mayor sentido. A su vez, este supuesto se funda en un presupuesto: que el más adecuado desarrollo de la capacidad lógica está vinculado al desarrollo de la imaginación (es decir, que los usos imaginativos del pensar -como escribir un poema o un cuento, como soñar, como la simple capacidad de examinar posibilidades y especular- son esenciales para el desarrollo de una capacidad de razonamiento sistemático y consistente). Creo que este supuesto es válido -y creo que también Lipman lo formularía así- si se concede a su vez el argumento inverso: que el adecuado desarrollo de la imaginación implica y exige un amplio desarrollo de la capacidad de razonamiento lógico. Tanto la lógica como la reflexión ética, tanto la política como la poesía son asuntos en donde se debe dar una adecuada combinación de lógica e imaginación. Desde luego, esto no es un simple supuesto general de la composición de $\mathrm{DH}$, sino que opera y está presente en su trama narrativa de diversas formas. Destacaré sólo una de ellas: el recurso a los sueños como formas de explorar problemas o de poner a prueba hipótesis, como ocurre en el "soñar despierto" de Elisa (cap. 3, pp. 23-24) y en el sueño de Toño con las cuatro posibilidades (cap. 13, pp. 105-107). Este "recurso al sueño" es una forma de extender el uso de las reglas más allá de sus usos habituales para ver hasta dónde llegan los límites de su aplicación; una "ruptura" con "las reglas del pensar" que nos permite ver los límites específicos del discurso lógico.

6. El énfasis puesto en los "actos mentales": DH es un texto para leer "entre líneas", en el que se debe poner suma atención a los términos usados en cada momento, pues ninguno de ellos es gratuito o accidental. Aunque muchas nociones lógicas centrales (como la de "relaciones transitivas") se presentan en términos coloquiales propios de nuestro lenguaje ordinario (como "relaciones que pasan" o "relaciones que se transmiten"), cada término está escogido con sumo cuidado, atendiendo a que ese término ayude a entrar al lector en un determinado problema filosófico (lógico o de otro tipo). Es especialmente importante poner cuidado en el uso que hace Lipman de ciertos verbos como pensar, creer, explicar, describir, replicar, preguntar, objetar, asentir, querer decir, pensar para sí mismo, etc. (en ocasiones esos verbos incluso aparecen subrayados en el texto o con cursivas). El uso de tales verbos es absolutamente intencional y explícito, pues ellos señalan y describen actos mentales específicos que podrían ser objeto de una indagación filosófica más detenida. El examen de los "actos mentales" (es decir, de aquello que hacemos al discutir, preguntar, reflexionar, estar de acuerdo, etc.) es uno de los caminos más adecuados para suscitar el ejercicio de la reflexión filosófica en los niños y jóvenes. 


\section{La relación de la lógica con otros problemas fillosóficos}

Hemos dicho hasta aquí que DH es una novela, incluso un "programa", de lógica formal. Esto es cierto, pero no lo es todo. Y esto por dos razones básicas. En primer lugar, porque, aunque la lógica presente en $\mathrm{DH}$ es básicamente la lógica formal clásica (aristotélica) ${ }^{8}$, en realidad en este texto hay mucho más que la lógica clásica. Por ejemplo, hay también, algunos elementos de "lógica de relaciones" (como los ya señalados en los caps. 7 y 8), de lógica inductiva (especialmente en el cap. 5) y de lógica del razonamiento moral (cf. caps. 9 y 10). En segundo término, DH no es sólo un programa de introducción al razonamiento lógico (deductivo), sino algo más: una introducción a la investigación filosófica (no es gratuito, por ello, que el manual del profesor que acompaña a esta novela tenga por título precisamente "Investigación filosófica"). En tal sentido, podemos afirmar que en $\mathrm{DH}$ aparecen casi todos los temas filosóficos básicos, como lo vimos en el punto anterior cuando intentamos describir la estructura teórica del texto.

Así pues, el "tema central" de DH es la lógica formal ("las reglas del pensar") y es en torno a él que se articulan todos los demás problemas filosóficos. La lógica, ya desde la antigüedad, fue considerada como la "puerta de entrada" básica a la filosofía, no sólo porque era concebida como la "propedéutica de todo saber" sino porque, en la enseñanza superior, e incluso en algunos casos en la educación básica, el entrenamiento en el razonamiento formal era el punto de partida de la formación de una persona culta (así lo era en la universidad medieval, y, en buena medida, lo sigue siendo en la tradición filosófica anglosajona).

En ese sentido, el programa FpN puede situarse dentro de esta tradición de educación filosófica. Por supuesto, no es la mera tradición lo que justifica la enseñanza de la lógica como el punto de entrada a la investigación filosófica en un programa como el de Lipman; más aún cuando es preciso justificar que este tipo de conocimiento sea desarrollado con muchachos alrededor de los doce años. Ahora bien, ¿cómo puede esto justificarse? A este respecto, Lipman insiste en dos puntos básicos:

a. Puesto que uno de los propósitos básicos de FpN es ayudar a los niños y jóvenes a pensar de una forma clara y ordenada, la lógica formal representa un ingrediente esencial para lograr este objetivo. Desde este punto de vista, Lipman pone el énfasis en ciertas cualidades mentales básicas que el trato

8 De allí precisamente el nombre dado por Lipman al protagonista (Harry Stottlemeier), nombre que, por lo menos en el original inglés, tiene una cierta semejanza con el nombre del gran filósofo griego. Aunque en algunas traducciones se han creado nombres que intentan, en español, conservar esa referencia (como Ari Stoteles en la traducción argentina y Aristeo Téllez en la traducción mexicana), me han parecido nombres demasiado forzados, por lo cual he preferido, en la adaptación que he hecho para Colombia, conservar el nombre original en inglés, que, a pesar de todo, resulta menos artificial que estas adaptaciones al español. Ello permite, además, no perder ciertas referencias históricas importantes que hay en el texto (como la que hay a la guerra del Vietnam en el capítulo 15), pues el padre de Harry aparece como un norteamericano que llegó a nuestro país hace algunos años, algo que suena más creíble que la creación de nombres en español excesivamente extraños y artificiales. 
con las reglas de la lógica formal puede proveer a los niños y jóvenes. Según él, el acercamiento a un sistema formal genera actitudes mentales como la consistencia (es decir, el esfuerzo por no incurrir en contradicciones), la consecuencia lógica (que es el resultado de manejar reglas que nos permiten hacer deducciones correctas, y esto, a su vez, nos hace más sensibles a las falacias o errores de razonamiento, y nos permite detectarlos más fácilmente) y la coherencia (entendida ésta como el hábito de pensar de un modo sistemático y unificado). De esta manera, el presupuesto en que se funda la idea de trabajar con las reglas de la lógica formal con los preadolescentes y adolescentes es que quien conoce las reglas básicas del razonamiento válido, y practica el ejercicio de hacer deducciones siguiendo reglas explícitas, participa de un sistema coherente. Así mismo, desarrolla el hábito de pensar de una forma ordenada y sistemática ${ }^{9}$.

b. Sin embargo, hay una razón más fuerte aún para empezar a trabajar la lógica formal con los jóvenes que están empezando a entrar en la adolescencia: es para ellos necesario, y constituye un elemento esencial de su "tránsito" hacia la vida adulta, el que se acostumbren a pensar en términos abstractos, formales. Es decir, que vayan más allá de su hábito de pensar las cosas siempre en términos concretos. Para ello, deben habituarse a usar el "lenguaje del pensamiento", es decir, no sólo a realizar operaciones mentales como las que siempre realiza cualquier persona, sino a tomar conciencia de cuáles son esas operaciones mentales que realizan; ello amplía inmensamente su poder de reflexión.

En la medida en que empiecen a tratar con este "lenguaje del pensamiento" que provee DH estarán en condiciones de alcanzar dos elementos básicos para su desarrollo cognitivo: en primer lugar, estarán en capacidad de pensar sobre su propio pensamiento, es decir, a realizar procesos metacognitivos, lo que, a su vez, les permita generar un cierto grado de autoconciencia respecto de su propio aprendizaje. En segundo término, en la medida en que puedan aprender a pensar en términos de proposiciones y de argumentos regidos por estrictas reglas de validez, lograrán liberar su capacidad de pensar de la inmediatez de los datos sensibles, lo cual es un punto de apoyo fundamental para la consolidación de su capacidad de pensar de forma autónoma en la medida en que les permite pensar en términos de posibilidades imaginadas, de supuestos subyacentes y de consecuencias previsibles.

Las razones anteriormente mencionadas son precisamente las que justifican la importancia de trabajar de un modo cuidadoso DH con los preadolescentes. Lo que se espera de un trabajo como el que puede realizarse con este texto es que estos jóvenes, en la medida en que se habitúen a razonar de la forma ordenada y sistemática en que lo hacen los personajes de la novela, empiecen a adquirir la capacidad para pensar de un modo abstracto. Sin duda este es uno de los

9 Sobre estos asuntos profundiza Lipman en el capítulo 11 de La filosofía en el aula (1992), especialmente entre las páginas 231-242. 
servicios más esenciales que hoy podemos prestar a las nuevas generaciones, especialmente tratándose de personas que están entrando en aquello que Piaget llamó "el estadio de las operaciones formales" (Piaget, 1981, pp. 94-99) ${ }^{10}$.

El énfasis que he hecho hasta aquí en la importancia de que los preadolescentes aprenden a razonar formalmente no debe engañarnos. La lógica formal tiene muchos límites. En primer término porque nuestras formas habituales de razonamiento no se rigen necesariamente por las reglas de la lógica formal. No razonamos la mayoría de las veces siguiendo reglas explícitas (a menudo lo hacemos, más bien, a partir de modelos, ejemplos, analogías, casos particulares, etc.), ni hablamos en silogismos. Cuando intentamos razonar sobre un asunto cualquiera recurrimos en la mayoría de las ocasiones a estrategias argumentativas que no tienen nada que ver con las reglas de la lógica formal11; aunque, por supuesto, hay principios generales de razonamiento a los que en principio no solemos renunciar si pretendemos pensar con claridad, como la máxima general de no incurrir en contradicciones.

En tal sentido, lo importante aquí no es simplemente "aprender reglas", pues el mero aprendizaje de reglas no es garantía de un mejor pensamiento (como el saber "reglas de ortografía" no es garantía de que escribamos correctamente); lo esencial es volvernos cuidadosos con nuestros procesos de pensamiento. Precisamente para ello, el ir descubriendo ciertas reglas que determinan la validez de nuestros razonamientos puede ser de inmensa ayuda. Esta "actitud crítica" ante el valor efectivo de la lógica formal como ayuda para el desarrollo de un mejor pensamiento se manifiesta a lo largo de $\mathrm{DH}$ de muchas formas distintas: en las dudas que manifiesta Toño respecto de su posible utilidad (cap. 2), en las conclusiones de Elisa en torno al campo de aplicación de estas reglas (sobre todo, cuando se da cuenta en su "sueño" que "las reglas de Harry" funcionan cuando están intentando explicar el mundo, pero no rigen lo que pueda ocurrir en su sueño, donde es posible que "todos los animales sean gatos"), en la prevención contra la obsesión de Harry y Toño de encontrar "reglas" en toda parte (manifestada especialmente por Elisa y Marcos), o en las propias conclusiones certeras a las que llega Elisa en muchas ocasiones de forma más bien intuitiva y sin tener que hacer uso de reglas explícitas de razonamiento.

Desde este punto de vista lo importante no es obsesionarnos con el aprendizaje o con la aplicación continua de reglas de validez del razonamiento. El descubrimiento y la permanente aplicación de las "reglas del pensar" deben, más bien, llevarnos (como ocurre con Harry, Toño y los demás personajes de la novela) a que nos hagamos más sensibles a los diversos contextos de conversación en que aparecen formas argumentativas que pueden resultar indeseables o

10 cf., pp. 94-99. Una explicación más completa de este asunto puede verse en el capítulo 5, "La elaboración del pensamiento. Intuición y operaciones", de su libro La psicología de la inteligencia (Piaget, 1989, pp. 133-170).

11 Un análisis muy valioso de las múltiples estrategias argumentativas que efectivamente usamos para resolver o plantear mejor diversos tipos de problemas es la que hacen Perelman y Olbrechts-Tyteca (1989) en su famoso libro Tratado de la argumentación. La nueva retórica. Una versión más sintética de este estudio sobre las formas de argumentación se encuentra en El imperio retórico. Retórica y argumentación (Perelman, 1997). 
desafortunadas. Las reglas de la lógica formal que se pueden ir descubriendo a medida que se va trabajando con $\mathrm{DH}$ pueden constituirse en criterios de valoración de nuestros procesos de pensamiento, en elementos a partir de los cuales juzgar si el procedimiento a través del cual hemos elaborado una determinada conclusión es o no correcto. Lo importante no es razonar siempre según reglas, sino aprender a distinguir qué circunstancias exigen ese tipo de razonamiento y cuáles no. A este respecto, el siguiente comentario de M. Lipman puede resultar ilustrativo:

Sería un error suponer que la lógica formal por sí sola va a promover el pensamiento filosófico. Mientras que la lógica formal puede servir como un medio eficaz para ayudar a los niños y jóvenes a darse cuenta de que pueden pensar de una manera organizada, no nos da las claves para saber cuándo es útil y apropiado pensar de acuerdo con las reglas de la lógica formal y cuándo es simplemente absurdo. El pensamiento crítico sólo llega a ser pensamiento filosófico cuando es consciente de las limitaciones de sus propios patrones críticos. Y la lógica formal sola no nos proporciona semejante discernimiento (Lipman, 1992, p. 234).

Este comentario de Lipman contiene algunas precisiones importantes que no quisiera dejar pasar desapercibidas:

a. La lógica formal puede ser una buena "puerta de entrada" al pensamiento filosófico, pero no es el pensamiento filosófico por sí mismo, es decir, lo distintivo de pensar filosóficamente no es exclusivamente el saber razonar en términos proposicionales. Los matemáticos, por ejemplo, desarrollan, por la naturaleza propia de su disciplina, una capacidad inmensa para el razonamiento formal, y no son necesariamente por ello buenos filósofos. La capacidad de pensar en términos proposicionales es una de las cualidades esenciales de una mentalidad filosófica, pero no es ni la única ni necesariamente la más esencial.

b. De todas maneras la lógica formal será siempre esencial en la formación de una mente filosófica en la medida en que aporta algunas herramientas de pensamiento crítico que son insustituibles (por ejemplo, la posibilidad de examinar las relaciones posibles entre las proposiciones utilizadas en un argumentación, o la capacidad para realizar múltiples inferencias a partir de una proposición dada, o la de examinar la posible validez de una determinada forma de razonar a través de su traducción a la forma del silogismo, etc.). Sin embargo, el pensamiento crítico es sólo uno de los aspectos del pensamiento filosófico, que tiene otra serie de dimensiones (intuitivas, analíticas, reflexivas, creativas, solidarias) que, aunque, sin duda, la lógica formal puede efectivamente ayudar a potencializar, no constituyen su objeto específico de interés.

c. Por tanto, deben desarrollarse, junto con las estrategias de análisis que nos ofrece la lógica formal, otro tipo de estrategias ordenadas a generar pensamiento 
filosófico que vayan más allá de estas herramientas de pensamiento crítico. Dentro de tales estrategias (para el desarrollo de las cuales hay múltiples herramientas en los manuales de las novelas de $\mathrm{FpN}$ ) podemos destacar las siguientes: los ejercicios de razonamiento hipotético y contrafáctico, la exploración de conceptos a través de planes de discusión, los ejercicios orientados a clarificar y reforzar distinciones conceptuales, el análisis de situaciones morales, los ejercicios de escritura, la resolución de enigmas, etc. Todas estas estrategias sirven, tanto para complementar la formación de habilidades de razonamiento lógico, como para hacer uso de dichas habilidades en el análisis de problemas éticos, estéticos, políticos, metafísicos, etc.

d. Puesto que la lógica formal tiene un campo de aplicación limitado, el buen razonamiento debe cultivarse también, y de forma permanente, por medio del examen de problemas filosóficos "de contenido". En este sentido, los problemas filosóficos no son exclusivamente problemas de buen razonamiento. El buen razonamiento es la base a partir de la cual se analizan otro género de problemas que tienen que ver con la acción recta, con la comprensión de leyes explicativas de la experiencia natural, con la legitimidad de nuestras posturas políticas, con la formación en virtudes, con el examen de nuestras obras desde criterios estéticos de armonía, originalidad, etc.; pero encontrar una posición razonable en cuestiones de este estilo es mucho más que razonar correctamente sobre ellas. Es en este sentido que ha insistido siempre Lipman en la noción de "buenas razones" (que son oportunas, intuitivas y sensibles al contexto) como un complemento necesario de la lógica formal.

Con lo anterior entramos en una de las características esenciales de DH como un programa de formación en habilidades de razonamiento lógico: aquí la lógica formal se aprende como una de las herramientas básicas de la investigación filosófica; es decir, como algo que debe ser continuamente aplicado al examen de problemas filosóficos de diversa índole. El supuesto fuerte de un texto como éste es que el buen razonamiento (o el razonamiento correcto) es tan esencial a la hora de solucionar un problema de orden matemático como lo es a la hora de enfrentar un dilema ético, evaluar una postura ante un problema de orden social o político o escribir un cuento, un poema, un ensayo, o incluso una adivinanza o una fábula. Cultivando la capacidad de razonamiento lógico estamos preparando a nuestros estudiantes tanto para pensar mejor como para expresar ese pensamiento de una forma creativa en cuestiones prácticas como las decisiones morales o la capacidad de expresión escrita. Así mismo, tiene mayores posibilidades de desarrollar su imaginación (sea la imaginación moral, estética o política) alguien que razona de forma ordenada y sistemática $\mathrm{y}$, por tanto, manejando reglas de razonamiento que, según la circunstancia y necesidad, puede aplicar con rigor o "violar" de forma consciente para fines expresivos o creativos.

A lo largo de todo el texto de DH esto se puede percibir de múltiples maneras (y se percibe mejor, aun cuando uno mira la serie completa de textos que forman, además de éste, Elisa, Susy y Marcos). Un lector atento de DH podrá percibir 
fácilmente de qué manera "las reglas del pensar" son la clave esencial a partir de la cual pueden enfrentar de un modo razonable Harry, Toño, Elisa, Susy, Marcos y los demás compañeros de curso las cuestiones teóricas y prácticas que se les van planteando a medida que se desarrolla la historia. Es a partir de ellas que se examinan problemas éticos (caps. 9 y 10), cosmológicos (cap. 13), estéticos (cap. 13) o políticos (cap. 15). Para mostrar esto un poco mejor, me ocuparé a continuación de dos ejemplos tomados de pasajes de la novela.

El diálogo de Harry con su papá a propósito de "fumar cigarrillos" y de la guerra del Vietnam (cap. 15, pp. 125-127). El punto básico a partir del cual se genera esta conversación entre Harry y su padre es una pregunta simple: "Papá, ¿por qué fumas?". La respuesta que ofrece este último no satisface a Harry, pues parece que él estuviera preguntando si es que puede existir una razón que justifique fumar, mientras que la respuesta de su papá no ha dado ninguna razón al respecto, sino que sólo le ha dado un motivo para hacerlo ("me gusta"). Cuando Harry empieza a explorar un poco más a fondo la respuesta de su papá, comienza a buscar una causa que explique el hecho de que su papá fume; es entonces cuando su papá le cuenta que comenzó a fumar por la tensión que vivía cuando participó en la guerra del Vietnam. Hay aquí ya, de entrada, un problema filosófico complejo -el de la distinción entre razones, motivos y causas cuando se intenta dar cuenta de una acción o se pretende justificarla, uno de los problemas centrales de los que se han ocupado los filósofos morales de tradición analítica-. Éste es, sin duda, un problema tanto lógico como ético, que se genera especialmente por los múltiples significados que tiene un término como el "por qué" con el cual iniciamos ciertas preguntas. ¿Cómo debe abordarse un problema como el planteado por Harry a su padre?

En primer término, exige un examen de relaciones causa-efecto: ¿qué es lo que causa qué? Eso es lo que hace Harry, siguiendo una estrategia semejante a la utilizada por Sócrates con el joven Eutifrón en el diálogo platónico que lleva el nombre de este último (Platón, 1997, pp. 213-242). Así, lo que parece estar planteando Harry a su padre son cuestiones como éstas: ¿¿Fumas porque te gusta o te gusta porque fumas?"; "¿Odiamos a otros porque peleamos con ellos, o nos peleamos con ellos porque los odiamos?". Estos asuntos pueden admitir múltiples respuestas y dejan, por ello, abierto el problema para su examen y discusión. De cualquier manera, éste es un posible ejemplo de cómo ciertas cuestiones de orden práctico (como el hecho de fumar o el significado que pueden tener las guerras entre los hombres) dependen a su vez de un correcto planteamiento de los problemas y del examen de las relaciones lógicas que existen entre los términos de la discusión en que estamos comprometidos.

La discusión sobre si el mundo tuvo o no un comienzo, y si fue creado por Dios (cap. 13, pp. 101-107). Aquí aparecen diversas afirmaciones en torno al origen del mundo, algunas de las cuales expresan una aparente contradicción existente entre ciertos enunciados científicos y religiosos. En vez de entrar en discusiones sobre creencias (que son muy delicadas) o en discusiones científicas (que son muy complejas), y después de que cada uno de los participantes ha puesto de 
presente los supuestos a partir de los cuales está argumentando, Toño propone el examen de la cuestión en torno al origen del mundo a partir de una estrategia que dice haber aprendido de un primo suyo: el análisis de posibilidades. Cuando todos han entendido en qué consiste la estrategia de razonamiento propuesta por Toño, empiezan a ensayar con ella en orden a examinar el problema que tienen planteado. Se encuentran entonces con el hecho de que, de las cuatro posibilidades dadas, sólo tres son posibles, porque una de ellas, la tercera, contiene una autocontradicción, lo que la hace lógicamente imposible.

La estrategia lógica elaborada para el examen del presente caso no está pensada para llegar a una solución única del problema planteado, sino para ayudar a identificar mejor las soluciones posibles; deja por ello abierta la posibilidad de discusión. Esta técnica del "análisis de posibilidades" es sumamente importante para plantear lógicamente cualquier problema, pues, aunque no nos conduzca a una solución (aunque, por supuesto, hay algo de solución cuando identificamos, por ejemplo, que una posibilidad no existe porque es autocontradictoria), nos permite plantearlo mejor en la medida misma en que, al ver las posibilidades que existen y examinar cada una de ellas por separado (como también al determinar que hay cosas que simplemente no pueden ser), podemos empezar a indagar mejor por sus supuestos teóricos y consecuencias prácticas. Esta es, por cierto, la misma técnica que con frecuencia tenemos que usar a la hora de intentar resolver ciertos enigmas por medio de un razonamiento lógico riguroso.

También en ese tipo de ejercicios podemos ver cómo el "análisis de posibilidades" es una de las estrategias más eficaces cuando queremos intentar encontrar una solución lógica a un problema por medio de un razonamiento ordenado que va pasando con suma precaución de premisa a premisa, haciendo sólo las deducciones que en cada instante se pueden hacer con absoluta seguridad, hasta llegar a la única solución lógica posible del enigma que se ha presentado para nuestro examen. En el pasaje que estamos analizando podemos ver cómo son ciertas estrategias de razonamiento (como ésta de "las cuatro posibilidades") la mejor ayuda para el examen de problemas teóricos tan difíciles de examinar (cómo éste a propósito del origen del mundo) sin caer en las creencias dogmáticas que buscan afirmarse sin considerar otras maneras posibles de comprender un mismo asunto.

Al final de la discusión anterior, y antes de que se duerman, Harry y Toño tienen una breve discusión sobre qué es lo que uno busca cuando piensa en un problema. A propósito de ello plantean dos hipótesis diferentes en torno al ejercicio del pensar que vale la pena examinar. Veámoslo en ese pasaje del final del capítulo 13:

Harry volvió a apagar la luz y, antes de acostarse en la cama, dijo, mitad para sí y mitad para los demás:

No tienes por qué estar siempre buscando respuestas. Es decir, puedes pensar sobre una cosa sin que tengas qué averiguar cuál es 
la respuesta correcta. Hay muchas maneras distintas de mirar las cosas y de pensar en ellas. Pero supongo que tienes que descubrirlas por ti mismo. Te enseñan que sólo hay una manera de pensar, y luego descubres que hay muchas otras maneras que pueden ser igual de buenas. Me gustaría descubrir todas las maneras de pensar posibles.

-A mí me interesa -replicó Toño- descubrir la manera correcta de pensar.

Harry hubiera querido decir "Y a mí también", pero ya estaba medio dormido (p. 106).

A esta altura de la novela se hace ya explícito lo que un lector atento de ella seguramente ha captado ya desde capítulos anteriores: que, aunque Harry y Toño son, junto con Elisa, los más activos a la hora de comprometerse en la búsqueda de las reglas del pensar, los anima a ello pretensiones diferentes. Toño busca siempre una regla que luego pueda aplicar a nuevos casos, a Harry le interesa el acontecimiento mismo del pensar en sus diversas manifestaciones. De esta manera, a Harry le interesa el pensamiento en general como una forma de plantear e indagar problemas (por eso no le preocupa tanto llegar a respuestas o a soluciones correctas, sino que lo que le anima es el deseo de descubrir todas las posibles formas de pensar en un problema); mientras que a Toño le anima la idea de descubrir una forma correcta de pensar siguiendo reglas que le permitan llegar a soluciones correctas por medio de métodos públicos y pasos explícitos.

Estas distintas maneras de enfocar el problema del uso del pensamiento pueden ser interpretadas como una forma de distinguir entre el pensamiento científico (orientado a resolver problemas) y el pensamiento filosófico (orientado a explorar esos mismos problemas en orden a su mejor planteamiento). Sin embargo, creo que pueden ser entendidas también en un sentido más amplio, como la base a partir de la cual podemos distinguir entre diversas formas de "pensamiento superior" que aparecen, encarnadas en distintos personajes de la novela, a lo largo de toda la trama de DH. Este asunto de las diversas formas o estilos del "pensamiento superior" (es decir, de un pensamiento que es sustancialmente mejor que nuestras habituales formas de pensamiento) es uno de los puntos centrales de este texto, y a su análisis nos dedicaremos a continuación.

\section{Las "formas del pensamiento superior" en DH}

El problema planteado por Harry y Toño al final del capítulo 13 es un asunto que está latente a lo largo de toda la trama de la novela y que recorre todo el texto. Podríamos formularlo de la siguiente manera: ¿hay muchas formas posibles de pensar o hay sólo una forma correcta de pensar? El punto central del asunto está en cómo habremos de entender la " $\mathrm{o}$ " que contiene la anterior pregunta. La conjunción "o" puede tener un doble sentido tal como la usa en español: puede 
ser incluyente o excluyente. Aunque, en lógica formal, se suele considerar básicamente el sentido excluyente de este término de enlace entre proposiciones (si digo "Vas al parque o al cine", debe entenderse que, si voy al parque, se excluye la posibilidad de que vaya al cine; $y$, si voy a cine, se excluye la posibilidad de que vaya al parque), en la anterior pregunta seguramente debemos entender la "o" en sentido incluyente. Es decir, no debemos suponer que el hecho de que existan múltiples formas posibles de pensar excluye el hecho de que exista una forma correcta de pensar (el propio Harry -nos dice el narrador al final del pasaje previamente citado- hubiera querido decir que a él también, como a Toño, le interesa la forma correcta de pensar).

En este sentido, DH es tanto un modelo de indagación sobre el pensar y sus múltiples formas o estilos como un modelo de pensamiento correcto (que es el que representa especialmente Toño, interesado en que todo lo que pensemos sea el fruto de reglas que pueden ser explícitas; en este caso, las reglas de la lógica formal). Así pues, no debemos plantear nuestra pregunta del párrafo anterior como un dilema que nos exige escoger una única opción y nos obliga a desechar la otra. Tan válido es explorar las múltiples formas de pensamiento superior que podemos desarrollar (como lo expresa Harry al decir que le gustaría "descubrir todas las formas de pensar posibles") como intentar determinar cuáles son las reglas generales a que debe ajustarse el pensamiento correcto (como lo afirma Toño).

Creo que lo que intenta destacar Lipman con este pasaje es que hay maneras distintas de situarse ante el problema del pensar. En el pasaje que vengo comentando aparecen por lo menos dos de ellas: 1) buscando las reglas que determinan un acto cualquiera de pensamiento (lo que hace Toño, que busca siempre reglas) con el fin de alcanzar, a través del examen, comprensión y sistematización de dichas reglas, soluciones efectivas a los problemas que desencadenaron su proceso de investigación; llamaré a esto una perspectiva resolutoria, ya que busca clarificar cómo construimos un conocimiento verdadero y cómo sabemos que efectivamente es verdadero. 2) Hay una segunda perspectiva en la cual se examinan los problemas mismos, ya no tanto a partir de reglas, sino, más bien, para establecer su significado e implicaciones posibles (es esto precisamente lo que hace Harry todo el tiempo; a él no le interesan sólo las reglas, le interesan los problemas mismos y se compromete a fondo en su investigación). Llamaré a esto una perspectiva problemática, pues se caracteriza por ahondar en el problema mismo, antes que en solucionarlo, examinándolo a la luz de sus supuestos, consecuencias, modos de argumentación, etc.

Creo, además, que, a medida que la trama de DH se desarrolla va apareciendo aún una tercera forma de situarse ante el pensamiento, representada especialmente por personajes femeninos como Susy, Ana María y María Fernanda. Me refiero a lo siguiente: 3 ) se trata de pensar desde una perspectiva en la cual no importan tanto los problemas o sus soluciones teóricas cuanto la forma como el pensamiento nos ayuda a modelar la realidad, sea ésta la realidad física, simbólica o social (en este caso el pensamiento se materializa en obras, que pueden ir desde los inventos tecnológicos, hasta las teorías y las obras de arte de todo tipo y las 
formas de organización social). Llamaré a esto una perspectiva creadora de realidad, puesto que se trata de una forma de situarse ante el pensamiento en la cual éste busca formas específicas de modelamiento o transformación de la realidad a través de la creación de instrumentos, símbolos, teorías, formas de convivencia social.

Estas tres formas de situarse frente al pensamiento, o, como también las he llamado, "perspectivas" (resolutoria, problemática y creadora de realidad) dan lugar a por lo menos seis formas distintas de pensamiento superior que, de distintas maneras, se van desplegando a lo largo de toda la novela. Cada una de estas "formas de pensamiento superior" se encarna, además, en personajes específicos de la novela DH que la representan de una forma peculiar. Aunque toda forma de clasificación resulta reduccionista, pues no puede dar cuenta de múltiples matices, presento a continuación un cuadro preliminar sobre estas formas de pensamiento superior tal como, en mi concepto, aparecen a lo largo de $\mathrm{DH}$ representadas en distintos personajes de la novela. Después de presentar dicho cuadro tendré que entrar más a fondo a caracterizar, a partir de la novela y sus personajes, cada una de dichas formas de "pensamiento superior".

Tabla 1. Las "formas del pensamiento superior" en DH

\begin{tabular}{|c|c|c|c|}
\hline $\begin{array}{l}\text { Perspectivas del } \\
\text { pensamiento } \\
\text { superior }\end{array}$ & $\begin{array}{c}\text { Formas del } \\
\text { pensamiento } \\
\text { superior }\end{array}$ & $\begin{array}{c}\text { Personajes que } \\
\text { representan o } \\
\text { tipifican estas formas }\end{array}$ & $\begin{array}{l}\text { Características generales } \\
\text { de estas perspectivas }\end{array}$ \\
\hline \multirow[b]{2}{*}{ Resolutoria } & $\begin{array}{l}\text { Pensamiento } \\
\text { analítico }\end{array}$ & Toño & $\begin{array}{l}\text { Orientado a la solución de } \\
\text { problemas. }\end{array}$ \\
\hline & $\begin{array}{l}\text { Pensamiento } \\
\text { intuitivo }\end{array}$ & Elisa & $\begin{array}{l}\text { Se desarrolla como una } \\
\text { habilidad básicamente } \\
\text { individual }\end{array}$ \\
\hline \multirow{2}{*}{ Problemática } & $\begin{array}{l}\text { Pensamiento } \\
\text { crítico }\end{array}$ & Marcos & $\begin{array}{l}\text { Orientado al planteamiento } \\
\text { de problemas. }\end{array}$ \\
\hline & $\begin{array}{l}\text { Pensamiento } \\
\text { reflexivo }\end{array}$ & Harry & $\begin{array}{l}\text { Se desarrolla en un ambiente } \\
\text { comunitario y dialógico }\end{array}$ \\
\hline \multirow[t]{2}{*}{$\begin{array}{l}\text { Creadora de } \\
\text { realidad }\end{array}$} & $\begin{array}{l}\text { Pensamiento } \\
\text { creativo }\end{array}$ & Susy, Ana María & $\begin{array}{l}\text { Se construye y expresa por } \\
\text { medio de obras que modifican } \\
\text { nuestras formas de producir, } \\
\text { nuestra sensibilidad, ideas y } \\
\text { formas de convivir. }\end{array}$ \\
\hline & $\begin{array}{l}\text { Pensamiento } \\
\text { solidario }\end{array}$ & María Fernanda & $\begin{array}{l}\text { Se desarrolla a través de } \\
\text { diversas formas de interacción } \\
\text { individuo-sociedad }\end{array}$ \\
\hline
\end{tabular}

Fuente: Elaboración propia

El cuadro que acabo de presentar requeriría, sin duda, de muchísimas explicaciones adicionales, que no es posible hacer en estos "apuntes". La clasificación que aquí elaboro podría ser objeto de amplia discusión. Sin embargo, debe tomarse no más que como una primera y posible diferenciación de estas formas de pensamiento superior $\mathrm{y}$, especialmente, como una forma de clarificar 
los diversos perfiles y estilos cognitivos que se pueden observar en algunos de los principales personajes de $\mathrm{DH}$. Lo que podré hacer por ahora no es más que una primera caracterización, muy general, de cada una de estas formas de pensamiento superior, referidas de una forma específica a personajes del texto. Corresponde a cada uno examinar, a partir de la lectura personal de la novela, hasta qué punto es adecuada esta caracterización.

Para empezar, conviene advertir que estas seis formas de pensamiento superior que aquí presentaré no son seis modos de pensamiento distintos (lo cual sería desconocer la unidad del pensamiento), sino modos distintos de conducir el pensamiento para alcanzar fines diversos. Por ejemplo, en algunos casos intentamos solucionar un problema siguiendo pasos específicos y reglas explícitas (pensamiento analítico), mientras que en otros procedemos "a saltos", lanzando propuestas de solución que se basan en una "mirada de conjunto" de problemas con los que poseemos un cierto grado de familiaridad (pensamiento intuitivo). En otras situaciones, nuestro esfuerzo por comprender el problema implica la construcción de criterios para su examen (pensamiento crítico) o una consideración más detallada de los supuestos, implicaciones y formas de argumentación que hay en él (pensamiento reflexivo). Hay otras ocasiones en que nuestro pensamiento procede creando realidad, que bien puede expresarse en obras de distinto estilo (tecnológicas, artísticas, etc.) (pensamiento creativo) o puede implicar situarse en la perspectiva de otros para "cuidar" de su bienestar o para promover formas de convivencia y reciprocidad (pensamiento solidario).

Aunque aquí hablaremos de cada una de estas formas como un modelo general (encarnado, por supuesto, en personajes específicos de DH) no debemos suponer en ningún caso que se trate de modelos que se den "puros" en sujeto alguno; aunque haya algunos individuos que podríamos calificar de "intuitivos más que analíticos", "creativos más que reflexivos", "solidarios más que críticos", etc., todos tenemos la posibilidad de desarrollar adecuadamente cada una de estas formas de pensamiento superior, aunque, por supuesto, por razones tanto de inclinaciones naturales como del ambiente en que vivimos y nos formamos, tendamos a desarrollarnos más en una perspectiva que en otra. Por tanto, no se trata de clasificar a las personas, o incluso de rotularlas, bajo uno sólo de estos estilos cognitivos, sino de comprender la gran variedad de estilos cognitivos que, por una parte, existen ya en nuestros alumnos, y, por la otra, podríamos ayudar a desarrollar en ellos.

Ademas, conviene advertir que ninguna de estas formas puede cultivarse por separado de las otras, y que precisamente unas y otras formas se requieren y se refuerzan. Así, por ejemplo, las posibilidades creativas están en muy clara conexión con el desarrollo de las capacidades analíticas, al igual que estas últimas tienen un claro vínculo con la capacidad de pensamiento reflexivo; o una cierta dosis de pensamiento intuitivo se requiere para el desarrollo de habilidades de pensamiento creativo. Aunque a continuación haremos una descripción por separado de cada una de estas formas de pensamiento superior, debemos 
insistir en que es precisamente la interacción entre todas ellas -como sucede precisamente a lo largo de todo $\mathrm{DH}$, en donde todas estas formas de pensar el mundo entran en permanente diálogo- lo que puede garantizar las mejores posibilidades de desarrollo para cada una de ellas.

El pensamiento analítico se caracteriza por el intento de descomponer un problema en sus partes constitutivas, de forma que, mediante un examen detallado de sus partes y de las relaciones existentes entre ellas, se encuentren perspectivas de solución al problema planteado. En este caso, para lograr alcanzar una solución se siguen métodos aceptados por todos y reglas que son explícitas. Este tipo de pensamiento se desarrolla dando un paso cada vez y posee una conciencia bastante amplia de la información que maneja y de las estrategias de solución que está utilizando, que incluyen en la mayoría de los casos reglas de razonamiento deductivo, algoritmos matemáticos o incluso diseños de investigación experimental. La gran ventaja de esta forma de pensamiento es que suele alcanzar los fines que se propone y lo hace con una amplia conciencia de los procedimientos involucrados en su proceso de solución. La observación detallada, la capacidad de cálculo y el buen ejercicio deductivo son características propias de los buenos pensadores analíticos. En DH el personaje que mejor representa esta forma de pensamiento, sin duda, es Toño, el amigo de Harry, que se caracteriza por ser el mejor estudiante de matemáticas y que, siempre que se presenta un problema, posee la habilidad para descomponerlo en sus elementos esenciales y para hallar soluciones rápidas y eficientes para él. Además de su gran capacidad para las matemáticas, Toño se fascina con el análisis del lenguaje, al que compara con un reloj que puede ser desarmado en todas sus piezas para comprender mejor su estructura (cf. p. 24). Esta tendencia analítica de Toño lo hace a veces un poco escéptico ante ciertas conclusiones, especialmente ante determinados juicios de Elisa, que, aunque certeros, podrían resultar demasiado aventurados, como ocurre con el robo de la cartera de su compañera Juanita en el cap. 16.

El pensamiento intuitivo, aunque es también una forma de pensamiento que tiene por finalidad esencial la solución de problemas, recurre a una estrategia completamente diferente al análisis: cree fundamentalmente en el poder de la "intuición", es decir, de un cierto acto de aprehensión inmediata de un objeto que le permite encontrar una solución rápida, y a menudo efectiva, al problema en cuestión. Aquí no hay "pasos", sino "saltos", que resultan posibles porque el pensador intuitivo, en tanto ha logrado desarrollar una gran familiaridad con los objetos con los que trabaja, puede percibir, de una sola mirada, la totalidad del problema, es capaz de ver de un modo inmediato la conexión entre todos sus factores y de encontrar una solución posible (muchas veces incluso la mejor solución posible) sin que, para ello, requiera dar todos los pasos que requiere el pensador analítico.

Para ello puede recurrir a las estrategias más inusitadas y, aunque sus respuestas no siempre sean certeras, se fundan en un conocimiento inmenso de las situaciones en que está involucrado. La "intuición" no debe entenderse aquí como una cierta "iluminación divina" o como "mera adivinación", sino como una 
forma de percepción cualificada que logran alcanzar determinadas personas (a menudo especialmente las mujeres) por la gran familiaridad con los hechos del caso. Los intuitivos, por supuesto, tienen la desventaja de que no siempre pueden ofrecer a otros una relación adecuada y suficiente de la forma como llegaron a su solución. El pensamiento intuitivo juega un muy importante papel a lo largo de todo $\mathrm{DH}$, pues, personificado por Elisa, está siempre a la base de todos los descubrimientos fundamentales que se van haciendo a lo largo del relato. A menudo es precisamente Elisa la que ve "el punto clave" de la cuestión $\mathrm{y}$, aunque no logre explicar siempre bien su intuición (con frecuencia Toño tiene que ayudarle a organizar sus intuiciones mediante el recurso al análisis), sus sugerencias e intuiciones son las claves que permiten casi siempre seguir avanzando en la exploración del problema de las "reglas del pensar". Por ello no es raro, que Harry casi siempre consulte especialmente con Elisa y Toño los principales descubrimientos que hace.

El pensamiento crítico se caracteriza por la tendencia a examinar los problemas a la luz de "criterios"12 en orden a perfeccionar los juicios que hacemos a propósito de circunstancias específicas. Por "criterios" debemos entender todas las diversas herramientas que utilizamos con el fin de darle mayor "peso" a nuestros juicios; así, por ejemplo, el reglamento escolar, la situación personal del alumno, su trayectoria como estudiante y otras cosas más son criterios que utilizamos para hacer un mejor juicio sobre su conducta en el colegio. El uso de criterios hace que el discernimiento que hagamos sobre una situación sea mucho más ponderado, en cuanto intenta tomar en cuenta todos los factores relevantes del caso; el que el pensamiento crítico se empeñe en considerar criterios variados y relevantes hace que éste sea esencialmente una forma de pensamiento autocorrectivo que, en vez de guiarse por principios absolutos, examina en cada caso las circunstancias particulares que determinan un contexto. El pensamiento crítico es, según la definición que nos propone el propio M. Lipman, un pensamiento que se rige por criterios, es autocorrectivo y sensible al contexto. Las diversas intervenciones de Marcos a lo largo de DH (y especialmente de las otras novelas de la serie, especialmente en aquella que lleva su nombre) son muestras valiosas de aquello en lo que consiste el pensamiento crítico: Marcos, cuando se plantea un problema, busca todo tipo de criterios para su examen $\mathrm{y}$, aunque a menudo las soluciones que ofrece no son las más adecuadas, sabe "poner el dedo en la llaga" de los problemas, como ocurre especialmente en la discusión que sostiene con Harry y su hermana María en el capítulo cinco de $\mathrm{DH}$ a propósito del sentido que tiene ir al colegio.

El pensamiento reflexivo se caracteriza por el esfuerzo de volverse permanentemente sobre sí mismo, sobre sus resultados, sobre sus supuestos, sobre sus propios procedimientos, para examinarlos con cuidado. Este examen permanente, esta "vuelta permanente sobre sí mismo", hace del pensador reflexivo alguien particularmente profundo. No va tan rápido como el pensador intuitivo, no es

12 "Crítico" no se debe confundir con "negativo". Ser crítico no es mostrar el aspecto negativo de una cosa o situación, sino saber utilizar "herramientas de juicio" (criterios) para hacer mejores juicios. Los términos "crítico" y "criterio" se forman a partir del verbo griego krino, que significa precisamente juzgar, discernir. 
tan certero en sus soluciones, como sí lo suele ser el analítico, pero, a cambio de ello, llega siempre al fondo de los problemas. El buen pensador reflexivo lo está examinando todo de una forma permanente: sus supuestos, sus consecuencias, el lenguaje que utiliza, la articulación lógica de su razonamiento; y, por ello, el ser reflexivo está continuamente haciéndose preguntas. Sin embargo, no se queda allí: intenta poner siempre en orden sus ideas, intenta controlarlas y ponerle una finalidad a todos sus descubrimientos y, especialmente, sólo ofrece sus conclusiones después de un detenido examen, análisis e investigación personal. Por supuesto esto suele hacerlo más lento, y lo obliga con frecuencia a dar diversos "rodeos" sobre las cosas. Harry es un buen modelo de pensamiento reflexivo: va siempre lento, a menudo se confunde; sin embargo, es persistente y está siempre elaborando y reelaborando sus ideas.

Es por lo anterior que siempre llega más lejos que los demás. Es él, por cierto, el que ve "el fondo" del problema (el de si hay unas reglas que rijan nuestros pensamientos y si podemos expresar nuestro pensamiento en términos de reglas que nos permitan hacer deducciones correctas) y logra, con su persistencia y con su esfuerzo de reflexión permanente, comprometer a todos sus demás compañeros en el asunto. Es cierto que Harry también está buscando reglas, como Toño, pero siempre va más allá de ellas. Él se pregunta por el significado del pensamiento (pp. 31-32), por la estructura de las proposiciones categóricas, por las relaciones existentes entre ellas, por los problemas de la relación entre causas y efectos, $y$, en general, por todas aquellas cosas que a muchos de sus compañeros aún pasan desapercibidas.

El pensamiento creativo se caracteriza por el hecho de su "materialidad", es decir, porque se expresa por medio de obras. Tales obras pueden ser de distinto estilo: inventos tecnológicos, obras de arte, teorías, etc. Pero el pensamiento creativo no sólo crea obras, sino que tales obras modifican de un modo u otro nuestra relación con el mundo: modifican nuestras formas de producción y comunicación (como lo hacen los diversos artefactos de la técnica), nuestras formas de percepción (como lo hacen las obras artísticas) o nuestras formas de explicar o comprender el mundo (como lo hacen ciertas teorías). Los únicos "creativos" no son los artistas, lo son también los inventores, los teóricos de distinto tipo e incluso, en cierto sentido, puede serlo el hombre común. Como forma de pensamiento, el pensamiento creativo se encarga también de producir un determinado tipo de juicios: juicios que se basan en criterios contrastantes y que a menudo resultan desgarradores y desenmascaradores, pues subvierten nuestra percepción del mundo y nuestra relación con él (esto es particularmente cierto, por ejemplo, en el humor, que suele ser una manifestación peculiar de pensamiento creativo).

En DH las formas de pensamiento creativo están ligadas esencialmente al arte (más que a la tecnología o la elaboración teórica) y se manifiestan especialmente en el caso de dos muchachas: Susy (que escribe poemas) y Ana María (que es pintora). Esto, en cierto sentido, es un límite, pues no hay, por ejemplo, un "inventor" entre los jóvenes de la novela; aunque también podemos decir que 
un personaje como Harry -que va descubriendo y estructurando una teoría lógica- es un creador de teoría. De todas maneras, en Ana María y Susy se ven dos casos específicos de pensamiento creativo en la medida en que se trata de dos personajes que sólo pueden pensar expresándose: la una a través de sus pinceles y de sus pigmentos y la otra a través de sus poemas. El capítulo 14 de $\mathrm{DH}$, en donde precisamente estas dos muchachas acuden al museo de arte, ofrece algunas referencias importantes para la comprensión del pensamiento creativo. En las otras novelas de la serie (y especialmente en Elisa y en Susy) estos modelos de pensamiento creativo se desarrollan y amplían mucho más.

Hablar de pensamiento solidario podría sonar algo forzado, pues no es lo propio del pensamiento "solidarizarse" con nadie. Sin embargo, desde la perspectiva de FpN se subraya muchísimo una cierta dimensión "ética" del pensamiento, que M. Lipman ha sintetizado en la noción de "caring thinking", que muchos suelen traducir por "pensamiento cuidadoso" o "pensamiento cuidante" y que, creo yo, expresa mejor la expresión "pensamiento solidario". Se trata de lo siguiente: un buen pensamiento es aquel que sabe ponerse en la perspectiva de otro (una de las máximas centrales del uso del entendimiento, según Kant), de ver las cosas poniéndose en su lugar para "cuidar" de él. "Cuida de ti mismo" y "cuida de los otros" constituyen dos máximas esenciales de la ética desde la antigüedad griega. Quien se guía por estas máximas y las incorpora en sus formas de pensamiento es alguien que se hace capaz de pensar siempre en términos de cuidado de los otros. La idea que mejor expresa hoy día esta capacidad de ponerse en la perspectiva de otros, y de intentar pensar desde allí, es la de solidaridad. Una caracterización más completa de esta forma de pensamiento superior es algo que aún está en construcción.

Sin embargo, en DH ya hay un primer modelo interesante de ello en la figura de María Fernanda, la muchacha negra que, al tiempo que defiende sus derechos (como mujer y como representante de una minoría étnica) desarrolla todos sus proyectos personales (como el llegar a ser abogada) desde la perspectiva del servicio que puede prestar a los demás; María Fernanda es alguien que, siempre que piensa en un problema, lo hace en términos de derechos y oportunidades sociales y que tiene por principio básico de todos sus pensamientos una regla general de justicia.

La caracterización anterior es muy general y, por ello, necesariamente muy inexacta. Rasgos específicos de cada una de estas formas de pensamiento superior descritas pueden encontrarse en otros personajes. Sin embargo, lo importante, decíamos, no es "rotular" los personajes de la novela, sino ofrecer "claves de lectura" para ésta. Además, intentar ampliar nuestra visión general de ese asunto que interesa tanto hoy a los educadores: el de la formación del pensamiento. Con frecuencia los maestros decimos simplemente que nuestros alumnos "no saben pensar"; sin embargo, nosotros mismos no tenemos muy claro qué tipo de pensamiento es el que debemos cultivar en ellos. Por ello a menudo intentamos desarrollar, por ejemplo, de una forma unilateral, habilidades específicas de pensamiento analítico, o de pensamiento creativo, 
descuidando otras dimensiones esenciales tanto para su vida como para su propio desarrollo intelectual. Enfatizamos con frecuencia el pensamiento analítico, el pensamiento crítico o el pensamiento creativo, sin embargo, descuidamos el desarrollo de sus habilidades de pensamiento intuitivo, reflexivo o solidario.

¿Cómo lograr, entonces, un desarrollo más amplio y coherente de estas diversas formas de pensamiento superior? Responder a esto no es asunto fácil. En cualquier caso, es esencial, por lo menos, tener identificadas esas formas de pensamiento que queremos desarrollar. En ello el trabajo con DH puede ser un buen punto de partida. Las diversas modalidades de pensamiento superior que allí aparecen, y que aquí hemos descrito de forma tan general, pueden ser iluminadoras para los propios jóvenes si, al trabajar con este texto, logramos que ellos se identifiquen con los personajes de la novela. No sólo Harry, sino también Elisa, Toño, María Fernanda, Marcos, Susy y todos los demás, podrían ser modelos cognitivos interesantes para nuestros jóvenes. Al maestro le corresponde, al tiempo que ayudar a los jóvenes a identificar estos distintos estilos y modelos cognitivos, enseñarles a los jóvenes con los que trabaja que sus propios estilos cognitivos son valiosos, y él sabe valorarlos, como lo hacen con cierta habilidad los maestros que aparecen en DH, especialmente el profesor Espinosa. Sin embargo, todo esto implica que el maestro, además de familiarizarse con el texto de la novela, comprenda las dificultades específicas que plantea el trabajo con un texto como éste en un aula de clase al tiempo que las alternativas que pueden tenerse a mano para superar tales dificultades. A ello le dedicaremos el último punto de los presentes "apuntes".

\section{Algunas sugerencias para el trabajo con DH en el aula de clase}

DH es un texto que presenta dificultades particulares cuando se emprende un trabajo sistemático con él en un aula regular. Por supuesto dichas dificultades resultan más complejas cuando no existe, con los niños con quienes se examina y discute este texto, un trabajo previo con el programa FpN. La condición esencial para que la experiencia con este programa resulte productivo es que los niños que participen en la experiencia se hayan venido constituyendo como una comunidad de indagación filosófica, en donde se plantean preguntas, examinan los supuestos de sus creencias, prevén las consecuencias de sus actos, se han empezado a acostumbrar a revisar sus razonamientos y al lenguaje en el que se expresan.

El desarrollo de estos hábitos propios de la indagación filosófica, desde luego, es a la vez una condición para el desarrollo de la experiencia y un resultado de ésta. Si estas cosas las han venido haciendo ya durante los años anteriores, hay un "terreno abonado" para que el examen y discusión de una novela que contiene un programa de lógica formal pueda dar mejores frutos. Sin embargo, en 
los casos en que esto ha ocurrido no dejan de presentarse algunas dificultades específicas con un texto que empieza a exigir a los muchachos que razonen en términos formales.

DH invita al joven que trabaja con él a que dé un salto cualitativo en el desarrollo de su pensamiento, pues pretende ayudarle a salir de las limitaciones propias de un pensamiento concreto y le ofrece la oportunidad para que empiece a pensar abstractamente en términos de proposiciones y "lenguaje del pensamiento". En uno y otro caso (cuando hay un trabajo previo en FpN y cuando se empieza por $\mathrm{DH}$ ), entonces, será conveniente hacer un trabajo previo de "aprestamiento" para que el acercamiento al texto y el tránsito hacia el pensamiento formal resulte menos tortuoso.

Antes de intentar ofrecer algunas ideas generales sobre lo que puede ser este "aprestamiento" conviene hacer presentes algunas de las dificultades más frecuentes que suelen darse cuando se trabaja con esta novela en un aula de clase. Dichas dificultades provienen de una triple fuente: unas obedecen a la naturaleza misma del texto, otras a limitaciones cognitivas específicas que suelen darse en el estudiante promedio de estos niveles y unas terceras a ciertos hábitos ya formados al interior de la institución escolar.

Dentro de las dificultades que provienen de la naturaleza misma de una novela filosófica como $D H$ podemos señalar las siguientes:

- Es un texto cuyo tema central es un problema abstracto, que no siempre atrae "de entrada" la atención de los estudiantes: el del pensamiento y sus reglas. Es éste un tema filosófico "duro" que no motiva tan fácilmente a los alumnos como puede motivarlos, por ejemplo, la discusión de problemas de filosofía "práctica", como los asuntos éticos, estéticos o políticos.

- Es probable que, por lo menos en algunos casos, los alumnos al comienzo se sientan "perdidos" sobre el tipo de lectura que exige la novela. Puede incluso que la historia, como pura historia, les llame la atención, lo cual, sin embargo, no implica que empiecen a comprender los problemas filosóficos que están presentes en ella, y mucho menos que empiecen a discutirlos y examinarlos por su propia cuenta.

- Incluso como mera historia, no es fácil para los muchachos comprender desde el comienzo -no es fácil ni siquiera para los propios maestros que trabajan con ellos - todos los recursos narrativos que están presentes en el texto; por ejemplo, la descripción de actos mentales o el modo como se procede en la construcción de las reglas básicas del razonamiento válido.

- Dado el carácter "abstracto" del problema con el que se está tratando, no se ve claramente la utilidad que pueda tener la discusión de un texto como éste. Eso seguramente a algunos muchachos no les preocupa, pues pueden descubrir simplemente que les resulta placentero discutir estos temas, pero habrá seguramente otros que querrán entender "para qué sirve" ese tipo de 
experiencia. Este problema de la "utilidad de la lógica", por otra parte, será algo a lo que necesariamente habrán de llegar, pues la trama misma del texto lo plantea. Por tanto, es necesario que el maestro que dirige la experiencia haga el esfuerzo por ofrecer oportunidades a los alumnos para que ellos descubran la utilidad de este tipo de reflexión.

- Tanto los personajes de la novela como los muchachos que leen y discuten el texto están situados en una etapa, la preadolescencia (entre los 10 y los 14 años aprox.), que se caracteriza precisamente por cierto grado de indecisión. Es ésta una etapa en donde, en cierto sentido, se sienten "desubicados", pues son niños que ya quieren ser grandes (aunque algunos todavía tengan cuerpos de niños) o grandes que en muchos aspectos todavía se comportan como niños (aunque algunos ya tengan incluso cuerpos de grandes). Esta situación ambivalente se refleja también en los personajes de la novela (algunos se comportan a veces caprichosamente e incluso hay niñas que todavía juegan con muñecas, al tiempo que se comportan con un cierto grado de "madurez" -sobre todo cuando entran a discutir temas tan complejos como el significado de la mente- que no suelen tener los niños "reales" de esa edad). Esto lo perciben fácilmente los muchachos que trabajan con el texto $\mathrm{y}$, aunque la historia pueda suscitar su interés, hay algunos de ellos que experimentan esta ambivalencia.

Todo lo anterior es sólo una muestra de la inmensa complejidad que encierra un texto como DH. Sin embargo, las dificultades que pueden presentarse al trabajar con esta novela no provienen exclusivamente de la novela misma. Pueden provenir también de ciertas dificultades cognitivas propias de los estudiantes, entre las cuales podemos destacar las siguientes:

- Las dificultades que, todavía en esta edad, tienen los niños y jóvenes para escucharse unos a otros. DH exige de quienes trabajan con él un nivel de argumentación mucho más cualificado y, sobre todo, una mayor atención a las formas de argumentación de los demás. Esto no es algo que se garantice fácilmente y que es preciso ir logrando a medida que se trabaje en el ámbito de una comunidad de indagación.

- Además de lo anterior, hay también un problema de comprensión de lectura, que es muy habitual en este nivel del sistema escolar (y que suele extenderse incluso más allá de él). Una buena lectura de DH supone que los jóvenes que trabajan con él hayan empezado a desarrollar una capacidad de lectura que les permita leer extrayendo significados relevantes de los textos que leen. No se trata sólo de que "entiendan" lo que leen (lo que, por supuesto, es un paso esencial), sino que empiecen a explorarlo y problematizarlo. El trabajo con la novela puede ayudar a desarrollar esta capacidad de "leer extrayendo significado"; sin embargo, es ésta también una condición esencial para un mejor trabajo con ella.

- A lo anterior se suma cierta dificultad para pensar en términos abstractos. Los niños de esta edad (no porque no sean capaces, sino porque ordinariamente 
no se les exige más fuertemente en su educación básica) suelen tener todavía dificultades para pensar sin tener que recurrir permanentemente a ejemplos o casos particulares, les cuesta mucho dar definiciones precisas de los términos que utilizan y, especialmente, todavía les resulta difícil revisar sus formas de razonamiento y pensar sobre sus propios procesos mentales. DH puede ser, si se trabaja adecuadamente con él, una excelente oportunidad para desarrollar en los jóvenes las diversas habilidades que se requieren para pensar abstractamente, es decir, para aprender a pensar en términos conceptuales.

- Hay alumnos que tienen, además de las dificultades generales que tiene cualquier muchacho de esta edad con la exigencia de pensar abstractamente, dificultades específicas para construir conceptos o pensar siguiendo reglas. Algunas de estas dificultades podrían identificarse con tiempo, antes de entrar a trabajar con el texto (por ejemplo, mediante algún test de razonamiento formal), de manera tal que, en la medida en que descubrimos dichas dificultades, podamos manejarlas mejor en el trabajo que se puede hacer en el aula con $\mathrm{DH}$.

A las anteriores dificultades señaladas, se suma un tercer tipo: las dificultades provenientes de ciertos hábitos adquiridos en el sistema escolar, entre las cuales podemos destacar las siguientes:

- Sólo hasta hace unos pocos años ha empezado a existir una preocupación seria por el desarrollo del pensamiento en la educación. Esta preocupación, sin duda legítima, se reduce en muchas ocasiones simplemente a buscar técnicas fáciles que ofrezcan resultados inmediatos. Sin embargo, DH no enseña precisamente "técnicas para pensar", sino que invita, más bien, a hacer a cada uno una reflexión personal sobre el hecho del pensar y a pensar sobre su propio pensamiento. Quien esté esperando que, con el simple hecho de trabajar con DH, se va a producir "como por arte de magia" una mejoría inmediata en los procesos de pensamiento de los alumnos puede llevarse una decepción.

- La educación hasta hoy prácticamente no ha tenido los procesos y reglas del pensamiento como uno de sus objetos de estudio básicos. Como lo señala Janeth en el capítulo 3 (p. 20) de DH, en el colegio se habla de todo lo demás: niveles anuales de lluvia, contaminación ambiental, adicción a las drogas, de las guerras, etc., pero poco se habla de cómo pensamos y del valor y significado del pensamiento en nuestras vidas. DH pone a los jóvenes a pensar sobre el pensamiento, $\mathrm{y}$, aunque el tema pueda resultarles apasionante, no es un tema fácil de tratar, sobre todo porque no es un asunto que se resuelva consultando un libro, sino precisamente pensando sobre lo que hacemos al pensar.

- Uno de los principales elementos de lo que Iván Illich llamó el "currículo oculto" de la escuela es precisamente éste: que se nos acostumbra a que hay que aprender lo que enseñan los maestros o lo que dicen los libros; es decir, que nuestro pensamiento no sólo no cuenta a la hora de aprender sino que, incluso, puede representar un obstáculo para el aprendizaje. "Limítese a lo que dicen los textos", "aprenda las cosas como son (es decir, como se las 
enseñó el profesor)" son frases que continuamente se escuchan en los ambientes escolares. El tipo de trabajo que se propone en $\mathrm{FpN}$, y especialmente con una novela como DH, va claramente "en contravía" de esto. Lo que aquí se propone es precisamente un aprendizaje reflexivo, donde se aprende no solamente lo que nos enseñan otros, sino donde el aprendizaje fundamental se logra a través de un proceso de síntesis de muchos factores de la experiencia que hacemos con ayuda de nuestra capacidad de pensamiento reflexivo.

- Además, el tipo de trabajo textual al que están habituados la mayoría de nuestros alumnos es el del análisis de los textos en donde se identifican ideas principales y secundarias, personajes principales y secundarios, secuencias de acontecimientos, etc., pero no problemas para la reflexión. Lo que pide el trabajo con una novela de $\mathrm{FpN}$ es algo muy diferente: no que los niños analicen el texto, sino que busquen en él sugerencias a partir de las cuales plantear problemas y explorar significados filosóficos. Al principio siempre hay dificultad con el hecho de que los niños y jóvenes con quienes se trabaja estos textos comprendan que el objeto del texto es ellos mismos y sus procesos mentales y no el análisis exhaustivo de una trama literaria.

La presentación de las anteriores dificultades (las más generales, por supuesto, dado que en la práctica concreta de aula se podrán identificar muchísimas más) no tiene por fin desanimarnos a trabajar con un texto como DH. Simplemente se trata de reconocerlas y saber que contamos con ellas. Ser consciente de esto es importante para que, en vez de crearnos falsas expectativas, podamos "pisar sobre terreno firme". Las dificultades no deben ser vistas exclusivamente como trabas o impedimentos; es preciso verlas también como oportunidades. En tal sentido habría que decir, por ejemplo, que lo importante es que DH se transforme en una especie de "reto cognitivo" para los jóvenes que leen y discuten este texto; no es un texto de fácil comprensión, pero, por ello mismo, puede generar en los estudiantes "conflictos cognitivos" que, adecuadamente manejados por los maestros que orientan este trabajo, pueden ser base para un aprendizaje significativo.

Igual que los obstáculos que tenemos que vencer cada vez que nos proponemos algo nos "elevan" por encima de nuestras preocupaciones inmediatas, las dificultades que hemos identificado o podríamos identificar no deben ser una razón válida para evitar la experiencia de trabajar con $\mathrm{DH}$. Más bien deben ser una invitación a que esa posible experiencia de aula con una novela como ésta se prepare cuidadosamente, evitando el mero ensayo y error, la improvisación fácil o la creencia errónea de que un texto, por sí solo, tiene el efecto mágico de modificar la conciencia y de transformarnos de un día para otro en personas críticas o en filósofos expertos. A este propósito conviene subrayar que $\mathrm{DH}$ no es simplemente un "texto de lectura" para una clase de filosofía, sino una historia a partir de la cual cada uno puede examinar su propio pensamiento ${ }^{13}$;

13 Con frecuencia me he encontrado con el hecho de que algunos profesores mandan a leer a sus alumnos $\mathrm{DH}$ como mandan a leer cualquier otra novela: para realizar un examen o "control de lectura" y discutirla en una o dos clases. Creo que quien hace esto no comprende el sentido que tiene este texto y la importancia de que se trabaje con él en el 
por tanto, lo esencial es poder leer el texto con cuidado, realizando la reflexión que en cada caso la lectura del texto puede propiciar.

Ahora bien, en la medida en que reconocemos que hay dificultades, sabemos también que hay que buscar mediaciones que nos permitan enfrentar y resolver favorablemente dichas dificultades. Tales "mediaciones" no son en todo caso soluciones mágicas, sino algunas alternativas que pueden ser viables en tanto las respalda una experiencia de trabajo previo. El sentido de estas mediaciones tal vez se comprenda mejor si hacemos aquí una comparación con lo que era la antigua medicina griega, la medicina hipocrática. Uno de los principios básicos que guiaba esta medicina era el de "dejar que obre la naturaleza"; para los médicos hipocráticos era claro que no eran ellos, sino la naturaleza, los que podían restablecer la salud de un enfermo, y que todo lo que ellos podían ofrecer a sus pacientes eran una serie de mediaciones (como la dieta, la observación de las estrellas, algunos remedios "caseros", etc.) que permitían restablecer el equilibrio corporal que se había perdido a causa de la enfermedad.

De una manera semejante, el maestro que trabaja con DH debe ser consciente de que todo lo que él puede hacer es ofrecer algunas oportunidades para que el aprendizaje filosófico que se puede realizar con este texto se realice efectivamente, pero que en ningún caso su labor como maestro consiste en "programar el aprendizaje de sus alumnos". Pensar es un proceso deliberado y no algo que pueda programarse mediante objetivos o logros prefijados. Al maestro le corresponde básicamente ayudar a propiciar el ambiente en el que los alumnos se sientan invitados a pensar y confiar en que no hay aprendizaje más efectivo y significativo que el que se realiza por medio de la reflexión propia y rigurosa. Debe confiar en que en sus alumnos "obra la naturaleza", es decir, debe confiar en los poderes cognitivos de los niños y/o jóvenes que se comprometen con la indagación acerca del pensar. Para que esos poderes cognitivos se realicen será necesario que el maestro utilice algunas mediaciones. Pero, ¿qué mediaciones? Señalaré aquí por lo menos cuatro mediaciones básicas:

1. Algunas estrategias que le permitan al maestro identificar los niveles de desarrollo cognitivo de sus estudiantes.

2. Mediaciones que les permitan comprender a los alumnos en qué consiste el trabajo en una comunidad de indagación.

3. Presentación de situaciones que inviten a los muchachos a razonar lógicamente y que les ayuden a descubrir el valor y el placer de hacerlo.

4. Estrategias que permitan introducir a los alumnos "en la mente" de Harry y sus compañeros, de tal manera que puedan comprender y aprendan a plantearse los problemas que ellos se están planteando.

ámbito de una comunidad de indagación filosófica como un punto de partida para generar una reflexión propia sobre los muchos asuntos que pueden estar sugeridos en esta historia. 
De cierto modo algunas de estas mediaciones pueden proveerlas el propio trabajo con el texto. Sin embargo, antes de empezar a trabajar con la novela es bueno desarrollar una fase de aprestamiento. Por "aprestamiento" se deben entender una serie de estrategias que deben generar las condiciones básicas para que los alumnos, a la vez que empiezan a constituirse como una comunidad de indagación filosófica, vayan descubriendo los problemas centrales que luego se trabajarán con el texto. Aunque las estrategias de aprestamiento existentes en $\mathrm{FpN}$ se han pensado fundamentalmente para el trabajo con los niños más pequeños, creo que el trabajo con $\mathrm{DH}$ requiere de un trabajo de aprestamiento específico, dada la característica propia de este texto y especialmente el hecho de que supone un tránsito hacia el razonamiento formal. Las herramientas de aprestamiento para este programa no están aún suficientemente consolidadas. Sin embargo, ofrezco para terminar algunas sugerencias de lo que podría ser ese "aprestamiento" tal como yo mismo he intentado desarrollarlo.

Antes de empezar a leer el texto de $\mathrm{DH}$ con los niños y/o jóvenes se pueden implementar algunas estrategias en tres fases básicas:

1. Una fase de introducción al razonamiento lógico, en la cual se les ayude a los muchachos a que descubran el valor del razonamiento lógico y las reglas que uno debe seguir para resolver problemas por medio de un razonamiento lógico estricto. Para ello, la estrategia básica puede ser la resolución de enigmas lógicos, que, al tiempo que les fascina, los obliga a que razonen siguiendo un orden estricto y haciéndose conscientes de las reglas que siguen en sus razonamientos. Se pueden utilizar para ello ciertos enigmas clásicos de la lógica, tomados de las obras de Raymond Smullyan, Irving Coppi y de otras fuentes. También se pueden buscar en Internet algunas páginas que contienen acertijos lógicos interesantes y sencillos.

2. Una segunda fase de desarrollo de habilidades de indagación, en donde se pone el énfasis en actividades como la observación, la pregunta, la descripción, la clasificación y comparación, el uso de ejemplos y analogías, etc. Aquí se puede recurrir a algunas técnicas de desarrollo de habilidades de pensamiento, como las que ofrece Margarita de Sánchez o las que se pueden encontrar en revistas como Juegos inteligentes.

3. Una tercera fase de transición al texto, en la cual, a partir de cuentos cortos (en donde Harry sea el protagonista) se les ayude a los muchachos a "entrar en la mente" de Harry y a comprender el tipo de problemas que él se irá planteando. Estos cuentos cortos, además de servir de transición hacia el texto de $\mathrm{DH}$, pueden ser la base a partir de la cual se proceda a la construcción de herramientas para la discusión filosófica. Yo mismo he escrito tres cuentos con ese fin, que intentan explorar lo que "pasaba por la mente" de Harry el día previo a la clase de ciencias con el profesor Espinosa en donde haría su primer descubrimiento. 


\section{Referencias}

Cohen, M., \& Ernest, N. (1990). Introducción a la lógica y al método científico. Buenos Aires: Amorrortu.

Coppi, I., \& Cohen, C. (2007). Introducción a la lógica. Buenos Aires: Amorrortu.

Dewey, J. (2010). Cómo Pensamos. Barcelona: Paidós.

Kohan, W., \& Waksmann, V. (comp.) (1997). ¿Qué es filosofía para niños? Ideas y propuestas para pensar la educación. Buenos Aires: Universidad de Buenos Aires.

Lipman, M. (1982). Harry Stottlemeier's discovery. Upper Montclair, NJ: Institute for the Advancement of Philosophy for Children.

Lipman, M. (1988). Philosophy Goes to School. Philadelphia: Temple University Press.

Lipman, M. (1991). Thinking in Education. New York: Cambridge University Press.

Lipman, M. (1992). On Writing a Philosophical Novel. En A. M. Sharp \& R. Reed (Eds.). Studies in Philosophy for Children. Harry Stottlemeier's Discovery (pp. 3-7). Philadelphia: Temple University Press.

Lipman, M. (1998). Pensamiento Complejo y Educación. (V. Ferrer, Trad.). Madrid: Ediciones la Torre.

Lipman, M. (2003). El descubrimiento de Harry: novela de lógica formal (2a ed.). (D. A. Pineda Rivera, Trad.). Bogotá: Beta.

Lipman, M., Sharp, A. M., \& Oscanyan, F. (1980). Philosophy in the Classroom. (D. A. Pineda Rivera, Trad.). Filadelfia: Temple University Press.

Lipman, M., Sharp, A. M., \& Oscanyan, F. (1984). Philosophical Inquiry: An Instructional Manual to Accompany Harry Stottlemeier's Discovery. Lanham: University Press of America.

Lipman, M., Sharp, A. M., \& Oscanyan, F. (1988). Investigación Filosófica. Manual del profesor para acompañar a El descubrimiento de Harry Stottlemeier. Madrid: Ediciones de la Torre.

Lipman, M., Sharp, A. M., \& Oscanyan, F. (1992). La filosofía en el Aula. Madrid: Ediciones de la Torre. 
Moriyón, F. G. (2011). Mattew Lipman: una biografía Intelectual. Crearmundos (9), 10-23.

Perelman, Ch., \& Olbrechts-Tyteca, L. (1989). Tratado de la argumentación. La nueva retórica. Madrid: Gredos.

Perelman, C. (1997). El imperio retórico. Retórica y argumentación. Bogotá: Norma.

Piaget, J. (1981). Seis estudios de psicología. Barcelona: Seix Barral.

Piaget, J. (1989). La psicología de la inteligencia. Barcelona: Crítica.

Piaget, J. (1998). La equilibración de las estructuras cognitivas problema central del desarrollo. México: Siglo XXI.

Pineda, D. (2004). Filosofía para Niños: el ABC. Bogotá: Beta.

Platón (1997). Eutifrón, en Diálogos I. Madrid: Gredos, Biblioteca Clásica Nº 37.

Splitter, L. J., \& Sharp, A. M. (1996). La otra educación: filosofía para niños y la comunidad de indagación. Buenos Aires : Manantial .

Vygotski, L. S. (2000). El desarrollo de los procesos psicológicos superiores. Barcelona: Crítica. 